\title{
36. THEORY OF STELLAR ATMOSPHERES
}

\section{(THÉORIE DES ATMOSPHÈRES STELLAIRES)}

President: R. N. Thomas.

Vice-President: R. Cayrel.

Organizing Committee: J. T. Jefferies, B. E. J. Pagel, J.-C. Pecker, V. V. Sobolev, A. B. Underhill.

SECRETARY: K. B. Gebbie.

PREFACE

This report has been prepared by the President in collaboration with the Secretary of the Commission. It is based on (1) material supplied by those members and associates of the Commission who responded to requests made in our circulars, (2) a bibliographical search intended to supplement the above material, (3) discussions with other interested persons, and (4) our own knowledge and impressions of the activity within the province of the Commission over the last three years. The report is divided into three parts. Part A is a scientific review aimed not at a comprehensive summary of all work but at a selective review of that which has, in our opinion, introduced some significant change in our concept of a stellar atmosphere or in the likely direction of future developments. Part B is a bibliography. It is this part of the report that is intended to provide an exhaustive summary of work done in the field over the last three years. To authors of those papers we have inadvertently omitted, we offer our apologies: neither were they sent to us nor did we locate them ourselves. Finally, Part $\mathrm{C}$ is an outline of the aims and activities of the Commission.

The decision to write the entire report ourselves, rather than delegate specific sections to other people, was aimed at providing a more coherent approach to the subject as a whole. Clearly, this represents only one of many possible approaches, and the form of the report is highly individual. The choice of what to include without encroaching on the fields of other commisions has become increasingly difficult. No longer can Commission 36 confine itself to theoretical models of stellar atmospheres. Empirical analyses that reveal inconsistencies in our theoretical assumptions must also be included, as must some consideration of solar phenomena, which are now recognized as being relevant to a wide variety of stars. Reference must also be made to those results of physics that are immediately applicable to the theory of stellar atmospheres. Inevitably, the selection has to some extent been arbitrary.

\section{PART A: SCIENTIFIC REVIEW}

\section{Introduction}

\section{a. A picture of a stellar atmosphere}

Our picture of stellar atmospheres is continually evolving. We are now beginning to realize that a stellar atmosphere in general comprises a number of regions, which differ widely in their properties. This has led to attempts to observe these properties and to understand why such regions exist. Is there some overall pattern in which the nature of chromospheres, coronas, expanding shells, cicumstellar dust, etc. may be better understood? Do all atmospheres have essentially the same regions, observed differences arising only from differences in the values of the state parameters, or do the number and variety of regions vary from one type of star to another?

Classically, the atmosphere was viewed simply as the outer boundary of the star, which was itself considered to be an isolated object. The atmosphere was assumed to be a single region, described by the same parameters as were used to construct the interior model: density, temperature, and chemical composition. Spectra were analyzed to determine the boundary values of these parameters, together with the range in these values from one type of star to another and from one region of the sky to another. Most studies of stellar atmospheres still fall into this category, progress 
being made chiefly through the availability of better observational data and the computation of more accurate single-region models.

The evolution away from this single-region model does not end with the construction of a socalled non-LTE region in which radiative processes are comparable to, or more important than, collisional processes in fixing the populations of the energy levels. Nor does it end with the recognition of solar-type chromospheres in solar-type stars, or indeed with the arbitrary inclusion of shells, dust, etc. in no overall pattern.

Rather, our picture seems to be evolving toward an atmosphere that is a transition region between the stellar interior and the interstellar medium. The properties of the interior are fixed, to a first approximation, by the characteristics of the star itself, independent of its environment. The stellar interior can be characterized as a storehouse of mass and energy, with only a small outward leak and a compensating inner replenishment. Its state - the concentrations of particles and the occupation numbers of the microscopic energy levels - can be described wholly in terms of the local concentrations of mass and energy, the fluxes of these quantities being irrelevant. This is a local thermodynamic equilibrium description in the broadest sense, using the methods of linear nonequilibrium thermodynamics. By contrast, the properties of the interstellar medium are fixed statistically by the fluxes of mass and energy from the background stars, and its state must be described in terms of detailed microscopic processes. The departure from local thermodynamic equilibrium is complete and must be described by non-linear, non-equilibrium thermodynamics. Viewed in this way, the stellar atmosphere represents a transition between these two states, which differ both in the set of parameters by which they are described and in the values of those parameters they share in common. In the classical, one-region picture of an atmosphere, this transitional aspect is neglected; the star is viewed as an isolated object, and the atmosphere is described in the same way as the interior. In the current multi-region picture, the star is treated as only quasi-isolated.

This current picture has evolved largely from two advances. One is the recognition that the detailed structure observable in the solar atmosphere is also required for the interpretation of integrated stellar spectra. The other is the recognition that apparently anomalous features observed in particular stars (e.g. P Cygni profiles, Wolf-Rayet spectra, UV, IR, and radio excesses) are simply exaggerated manifestations of physical effects more generally present. Together, they suggest that the 'symbiotic' appearance of stellar spectra is normal and reflects an atmospheric structure composed of several regions, differing in their physical properties.

To summarize, we might say that we are moving from a period in which research on stellar atmospheres focused on radiative loss of energy into a period in which it is recognized that mass flux may also be important in dispersing the energy generated in the stellar interior. Although the mechanical energy, together with the additional radiative energy associated with the mass flux, may indeed by small compared with the total energy produced, its effect on the structure of the atmosphere, and on the relation between the star and the interstellar medium, may well be essential. Just as early investigations underestimated the effect of radiation relative to mass (i.e. collisions) in fixing the state of the atmosphere and determining its spectrum, so they overestimated the role of radiation relative to mass in transporting its energy. If the inclusion of mass transport effects a significant change in the structure of model atmospheres and envelopes, perhaps we should also consider to what extent such changes can propagate downwards.

\section{b. A preliminary set of atmospheric regions}

An atmospheric region is distinguisted by the set of state parameters that suffices to give the local concentrations of particles and the occupation numbers of the energy states, both microscopic and macroscopic. The region is then described by specifying the range in the values of these parameters. Any attempt to divide the atmosphere into a set of regions is necessarily limited by our knowledge of the physical processes involved. We present here a preliminary set, which extends from the interior of the star to the interstellar medium, and within which each region is characterized by the behavior of one or more physical quantities. It is according to this scheme that we have then grouped the investigations discussed in the remainder of this report. 
Subphotosphere: That region in which the radiation field is fixed by its storage properties, and its transfer is by diffusion. Any transfer of mechanical energy is also by a diffusion-type process, characteristic of a storage region. Microscopic distribution functions are in LTE.

Photosphere: That region in which (a) the radiation field changes from quasi-isotropic to strongly anisotropic, (b) the energy transport is by radiation or quasi-static convection, and (c) there is hydrostatic equilibrium. We divide this region into two subregions, the collisionally controlled photosphere, in which collisions suffice to maintain local thermodynamic equilibrium, and the radiatively controlled photosphere, in which radiative processes play a significant role in fixing the occupation numbers of the energy states.

Chromosphere: That region in which the velocity fields change their character from non-dissipative to dissipative. Here it is the gradient of the mechanical energy flux that is important.

Corona: That region in which the mass flux becomes non-negligible in producing an enthalpy term, which must be included in the energy balance. Here it is the value of the mechanical energy flux that is important.

Circumstellar shells and nebulae: Those regions in which the interaction between stellar and interstellar material becomes increasingly important.

\section{c. Three approaches to determining state parameters}

Attempts to define and determine the values of the state parameters for a given atmospheric region may be divided into three broad categories: empirical, quasi-empirical, and theoretical. In the bibliography for this report, we group the various investigations according to the atmospheric region discussed and the approach taken.

\section{The empirical approach}

A set of observations, identified with a particular atmospheric region, is analyzed to give particle concentrations and occupation numbers, and an attempt is then made to find a set of paramvters by which to represent them. Solar eclipse studies, where the motion of the Moon's limb provides a way of locating a particular region, are the prototype of this approach. Eclipsing variables such as 31 Cygni present the closest stellar analogues. Since no one set of observations can cover all the relevant spectral regions, no atmospheric region can be analyzed wholly empirically, and care must be taken to distinguish those relations implicitly introduced. In the analysis of eclipse data, for example, the degree of ionization cannot be observed directly: any theoretical method introduced to determine it will depend critically on which lines and continua are included.

\section{The quasi-empirical approach}

Here a set of state parameters is adopted a priori, as are the values of some of these parameters throughout the atmosphere. The remaining ones are obtained by observation or theoretical assumption. The model is then used to predict all, or at least some, available observations and the distribution adjusted until an 'adequate' match is obtained. The uncertainty in this approach lies in the adequacy of the assumed set of state parameters and in the uniqueness of the predictions. What, for example, is the physical significance of the 'micro- and macroturbulence' parameters introduced ad hoc in every approach of this kind?

In practice, it is usually the distribution with height of electron temperature that is assumed, together with hydrostatic equilibrium and 'turbulence' parameters of some kind. The extensive work of Avrett, Cuny, and others on the solar atmosphere are examples of this type of approach. The category also includes the wide variety of models that adopt the LTE state parameters and the gray-body or radiative equilibrium temperature distributions. Currently, various approximations to non-spherically symmetric models are being explored by assuming a dependence of density on radius.

The approach provides the flexibility necessary to investigate the conditions for the production of, for example, emission lines in the absence of mechanical heating, fluorescent and laser effects, and various kinds of symbiotic effects. The latter include the appearance of apparently high and 
low excitation spectra in the same atmospheric region, of apparently incompatible spectra in different atmospheric regions of the same star, and of evidence for both an expanding atmosphere and a static atmosphere in different regions of the same star. The danger in this approach is that an ad hoc model be taken literally, without establishing under what conditions the assumed state parameters, and the ranges of these parameters, are physically consistent.

\section{The theoretical approach}

Here the model is based entirely on some set of theoretical assumptions, which, if it is to include different atmospheric regions, must include assumptions on their physical character. The extensive investigations of Auer and Mihalas probably represent the most systematic attempts to apply this approach to an atmosphere with two regions, one classical, the other in which the assumption of collision-dominated LTE is dropped. Attempts are now being made to represent chromospheres by three-region models based on theories of non-radiative energy dissipation. The extended-atmosphere models based on radiation pressure alone do not, however, include added atmospheric regions in the above sense.

The danger in this approach is that any given model be taken too seriously. Rather, successively less restrictive models should be computed until we have a complete picture of the microscopic processes that determine the state parameters under all conditions and can predict a set of atmospheric regions in accord with $A L L$ observations. The biggest difficulty is in knowing all the physical effects and the correct physical picture in which to include them.

\section{Subphotosphere}

In one class of models, mass motions are introduced for the sole purpose of reducing unstable radiative temperature gradients and are forced to vanish outside the unstable regions. With this approach, the emphasis has been on attempts to find a replacement for the mixing length theory of convection, which no one defends but everyone continues to use. With one exception, such attempts have so far been limited to investigations of the laboratory environment. The exception is the collaborative work of groups in New York and Nice (Latour, Spiegel, Toomre, and Zahn) in applying the non-linear theory to A-stars and the solar atmosphere. Preliminary results for A-stars confirm the mixing length result that there is negligible transport of convective energy but differ markedly from mixing length theory in the predicted velocities and the degree of convective penetration between upper and lower convective zones.

In a second class of models, the mass motions arising from the instabilities in the radiative temperature gradient are allowed to couple both with the mechanical energy dissipated elsewhere in the atmosphere and with the mass flux from the star. It is this approach that appears to have been most productive over the past three years.

(a) Studies of helium rich stars, in which one would expect convection zones to extend to stars of higher effective temperatures, indicate very high convective velocities. This is particularly true in white dwarfs, where, because the continuous opacity is low, the atmosphere includes regions of high density. Here the acoustic fluxes associated with such convection, as computed by the standard methods, become outstandingly high (cf. work by Nariai and Böhm's group in Seattle). Indeed the highest acoustic flux yet predicted, some $10^{11} \mathrm{erg} \mathrm{cm}^{-2} \mathrm{~s}^{-1}$, has been obtained by Böhm's group, treating this problem. Such results raise the interesting possibility of a chromosphere in a collisionally controlled atmospheric region.

(b) A result that apparently depends on magnetic fields leads to the conclusion that the mass flux in a stellar wind must be independent of magnetic fields. That the magnetic field, and hence rotation, decay with age is suggested by (1) the Skumanich-Wilson study of the relation between stellar age and $\mathrm{Ca}^{+}$emission, together with (2) the correlation, in the Sun, between $\mathrm{Ca}^{+}$emission and surface magnetic fields, and (3) the idea that surface magnetic fields arise in the coupling between convection and rotation. The detailed results suggest a linear relation between field strength and rotational velocity. Durney has shown that the momentum removed by the solar wind could 
not give such a linear relation if the mass loss depended on the magnetic field. Thus the magnetic field cannot be responsible for the mechanical energy required for the solar wind.

This result bears on the relation between the existence of a chromosphere and corona following from Praderie's suggestion that mass flux is a necessary condition for a chromosphere and dissipation of mechanical energy a sufficient condition. Durney's result suggests that either (1) the total mechanical energy dissipated in the chromosphere is independent of the magnetic field, or (2) the coronal temperature is independent of such dissipation, or (3) some combination of coronal temperature and density is independent of this dissipation.

(c) While great emphasis has been placed on convective motions as a source of acoustic flux for stellar chromospheres, the convective mode is only one of several unstable modes associated with a steep temperature gradient, and thermal instabilities only some among many possibilities in stellar atmospheres. Independent investigations by Leibacher and Ulrich of waves trapped in the photosphere and subphotosphere provide interesting alternatives. These waves are associated with the so-called opacity and $\lambda$ destabilizing processes in pulsating stars. In this formulation, the mechanical energy associated with a mass flux first appears in a stored configuration with a small leak corresponding to a combination of (1) the high frequencies not trapped by the solar minimum temperature and (2) the 'tunneling' exit in the upper photosphere. This brings into focus the parallel between the mechanical energy 'stored' in the sub-chromosphere, and the radiative energy 'stored' in the sub-photosphere, each propagating by 'diffusion' mechanisms. A further mechanism, suggested by Hearn for mechanical heating in hot stars, involves the interaction between acoustic waves and radiation pressure and may be of particular interest in Of and Wolf-Rayet Stars.

All these efforts suggest that we need no longer confine our attention to solar type chromospheres dependent on convectively produced acoustic flux for their energy and on magnetic fields for its dissipation.

\section{Collisionally controlled photosphere}

\section{a. Model atmospheres}

Of the large variety of LTE models in continuous production, perhaps the most interesting are those for cool stars and those in which the assumption of a plane parallel atmosphere has been dropped.

\section{Cool stars and opacity sources}

Here, as in many LTE models along the spectral sequence, the greatest advance lies in the inclusion of more realistic - and in some cases quite unexpected - opacity sources, as for example $\mathrm{C}_{2}{ }^{-}$(Johnson, Vardya). In considering the increasing number of line blanketed models, we must however remember that the assumption of LTE tends to overestimate the effects. Solar limb darkening results, for example, imply a strikingly low value for the temperature minimum (less than $3500^{\circ}$ ), if the spectra of molecules such as CO are assumed to be formed in LTE.

A systematic approach is now underway at the Institut d'Astrophysique in Paris to establish which opacity sources control the electron temperature in each part of a variety of atmospheres, both for LTE and non-LTE models. It is urged that those who compute model atmospheres output this data in a routine auxiliary table.

\section{Extended atmospheres}

An excellent summary of both extended and expanding atmospheres has been prepared by Böhm. Here we emphasize only the highlights and a few additional points.

With one exception, progress in this field has been made exclusively through the use of more accurate transfer solutions, the major advance being the introduction of a variable Eddington factor (Hummer and Rybicki). Gray models in which the opacity is assumed to vary as $r^{-n}$ reemphasize the forward peaking of $I_{v}(\mu)$ and the flatter distribution of $I_{v}(v)$.

Cassinelli, on the other hand, treats the nongray case and uses the momentum equation to compute an opacity distribution. He obtains the expected result that the total change in $T_{\mathrm{e}}$ between 
the boundary and $\tau=1$ is greater in the extended atmosphere, corresponding to the greater range in the dilution factor for the radiation field. He also finds the more surprising result that the boundary temperature is higher, and the boundary-temperature gradient lower, for non-gray than for gray models. Hummer and Mihalas obtain a similar result for a plane parallel model, which they attribute to heating in the Lyman continuum. We suggest, but have not yet demonstrated, that in both cases this result simply reflects the effect of electron scattering.

\section{b. Abundance determinations}

The vast majority of papers dealing with the collisionally controlled (LTE) photosphere are aimed at the determination of abundances. Previous Commission 36 reports have emphasized the importance of such results for interpreting differences in stellar spectra in terms of differences in stellar composition. That we do not do so here may be strongly criticized: all the papers we could find on the subject are included in the bibliography. Here, however, we elect to draw attention to the few investigations that explore the possibility of internal inconsistencies in analysis, which may signify that the derived abundances are open to question. As examples, we mention two such investigations, both solar. In the corresponding section on radiatively controlled photospheres, the problem is discussed in more detail.

Polarization studies at the Institut d'Astrophysique in Paris (Dumont, Pecker, Debarbat) provide an independent measure of electron density and thus of metal abundance. This work suggests higher values for the metal abundances than are normally accepted.

The increase in the accepted value of the iron abundance has been discussed extensively elsewhere. Suffice it to say that analyses of chromospheric and coronal lines, and of some forbidden transitions in photospheric spectra, appeared to give higher abundances than photospheric analyses of permitted transitions. It is now recognized that many of the photospheric discrepancies can be reduced by the use of more accurate $f$-values. Recent work on additional Fe I lines (Lites and Athay) suggests, however, that the inclusion of non-LTE processes may also be important in increasing the photospheric value. Similar work in Geneva (Müller, de la $\mathrm{Ráza}$ ) on $\mathrm{Li}, \mathrm{Be}$, and $\mathrm{K}$ also suggests that non-LTE processes may have a significant effect on abundance determinations. These studies belatedly confirm the heavily criticized non-LTE analyses of metals in the solar spectrum made a decade ago by the Pecker group at Meudon and Nice. Together, these results indicate that the boundary between the collisionally and radiatively controlled photospheres is deeper than had been previously accepted.

\section{Radiatively controlled photosphere}

\section{a. Abundance determinations}

The above results on metallic abundances in the Sun indicate that even relatively weak lines may be influenced by the radiation field. Inclusion of radiative processes tends to increase the determined abundances by about half an order of magnitude. This increase arises because the energy levels are photoionization dominated in a region of decreasing electron temperature and hence are underpopulated relative to their LTE values at the local electron temperature. Abundances determined on the basis of LTE will thus be too small.

By contrast, the assumption of LTE for the formation of the MgII lines in O and B stars leads to abundance determinations that are greater than normal. That the excessive strength of these lines can be accounted for by the inclusion of radiative processes with normal abundances has been shown by calculations of Mihalas.

The kind of differential effects that may occur even for lines of a given ion are illustrated by the work of Auer and Mihalas on the Her problem. They find that in B stars, lines in the blue-violet region of the spectrum may be analyzed as though they were in LTE, while those in the visual and red show very strong non-LTE effects. The direction of these effects depends on temperature and gravity, and far from being confined to strong lines, they are often strongest when the lines are very weak. These results arise in a general way because the lines are photoionization dominated and, as in nebular recombination lines, the size of the non-LTE effect is determined by the ratio of 
$\left[b_{\mathrm{L}} / b_{\mathrm{U}}-1\right]$ to [exp $\left.\left(h v / k T_{\mathrm{e}}\right)-1\right]$. Thus in the red, where $h v / k T_{\mathrm{c}}$ is small, significant non-LTE effects will occur even for relatively small $b$-ratios.

All these results emphasize the great caution with which LTE abundance determinations must be viewed, and together they illustrate the two competing effects that determine the direction of the non-LTE corrections. Because of the dominance of the scattering term in the expression for the source function, line depths and limb darkening, which depend on population ratios, will in general exceed predictions based on the assumption of LTE. The absolute populations, however, depend on the dominant mechanism - collisional or radiative - by which the levels are coupled with the continuum. Thus the actual abundance corrections may be positive or negative and may even differ for different lines of a given ion.

\section{b. Line profiles}

Perhaps the single outstanding problem in obtaining satisfactory line profiles is the necessity to introduce the arbitrary parameters of micro- and macro-turbulence. In solar models based on the CaII lines, for example, the inclusion of micro- and macro-turbulence has a greater effect than that of replacing the three-level by a multi-level atom or of replacing the simplest transfer solutions by the most sophisticated. (This does not, however, imply that LTE line profiles modified by velocity fields give the correct atmospheric model. In fact, they give the wrong sign for $\mathrm{d} T_{\mathrm{e}} / \mathrm{d} h$ ). Hence the problem lies not in the computation but in our physical understanding of the empirical phenomena. Various attempts have been made, with varying success, to account for these two parameters in terms of the velocity fields associated with chromospheric heating. However, since the same problem exists in predicting the MgII line profiles in $\mathrm{O}$ and $\mathrm{B}$ stars, this explanation would imply the existence of chromospheres, or some other explanation of 'turbulence', in hot stars. Indeed Mihalas has suggested that the behavior of HeII $\lambda 10124$ in emission in Of stars might be explained by the existence of a chromosphere, and many references have been made to the similarities between Of and WR classes. It is also interesting that the question of 'turbulence' arises as the common factor in discussions of (1) the Wilson-Bappu effect, (2) Kraft's use of the width of $\mathrm{H} \alpha$ as a luminosity indicator, and (3) the anomalous widths of line profiles in supergiants.

\section{c. Model atmospheres}

\section{Plane parallel atmospheres}

Since a model is a representation of a real stellar atmosphere, we must consider two questions: (1) to what extent do we know, and can we include, all the relevant physical processes, and (2) to what sophistication need we compute their effect?

To the extent that a limited number of ions and transitions suffice, the most complete representation of photospheres as defined in this report is probably given by the linearization method of Auer and Mihalas. Here a simultaneous differential correction is applied to all relevant state parameters at each point in the atmosphere, subject to all the photospheric conditions. Application of this method to $\mathbf{O}, \mathbf{B}$, and some A-stars demonstrates clearly the significant effect of non-LTE processes on the distribution of electron temperature and distinguishes the effect of lines arising from ions that are a major source of continuous opacity. Impurities, that is lines from ions not associated with continuous opacity, have thus far been treated mainly to infer their behavior in a given model rather than to study their effect on the model itself.

An algebraic approach, aimed only at a numerical accuracy compatible with the limitations of the physical representation, has been initiated by Gebbie and Thomas. Here the processes that affect the values of the states parameters are divided into two classes: those that arise from changes in the populations of the energy states, and those that arise from changes in the transfer quantities such as mass and radiation. The latter include the effects of impurities and mechanical heating. The effect of lines from ions associated with continuous opacity has in some cases been predicted algebraically by this method.

Among the photospheric models that have been computed, both LTE and non-LTE, perhaps 
the most controversial aspect is the effect of the lines. In the LTE models, this effect depends largely on the kind of statistics adopted for their distribution (cf. these by Peytremann and Querci). Athay's non-LTE approach to the Sun is also in a sense statistical, as are the non-LTE picketfence calculations of Mihalas and Luebke, which are based on the relative sized of a homogeneous set of lines, all characterized by the same collisional and continuous opacity parameters.

Thomas' original arguments that the effect of impurity cooling would be less in non-LTE than in LTE was based on the behavior of a single line at a fixed geometrical depth. Athay's counterarguments, on the other hand, concern the effect of the lines on the boundary temperature. It is therefore interesting to note that Peytremann's LTE calculations predict a boundary temperature for the Sun of some $4280 \mathrm{~K}$, in essential agreement with Athay's non-LTE calculation of $4330 \mathrm{~K}$. The latest empirical models (Eddy, McQueen, Noyes, Vernazza) do indeed lie near or slightly below these values, and unless molecular results (Lena, Hall) are strongly affected by non-LTE processes, suggested boundary temperatures may drop to $3800-3500 \mathrm{~K}$. The picket-fence calculations of Mihalas and Luebke show, however, that the effect of impurity cooling diminishes as the collision parameter decreases. Thus it should differ for collisionally and radiatively controlled lines, and it should vary with density from one luminosity class to another. At present, one can only say that the situation is uncertain. In discussions of line blanketing, however, care should be taken to distinguish the effect of impurity cooling from the heating and cooling effects of lines associated with ions that are a major source of continuous opacity.

\section{Expanding and extended atmospheres}

The Proceedings of the Munich Symposium provide a good overall picture of progress in this topic. Here we refer to them, remarking only as follows.

While the number of investigations of problems associated with moving atmospheres continues to increase, most of them still focus on the approach initiated by Sobolev: for sufficiently large velocity gradients, the value of the net radiative bracket (which, multiplied by the population of the upper level, gives the net rate of radiative transitions) is effectively that of a homogeneous finite slab. The form of the source function then becomes as simple as for a locally opaque configuration: in neither case does the radiation field appear explicitly in the expression for the source function, which is thus determined locally by the ratio of the source to sink term. In the locally opaque case, the sink term is the destruction probability; in the finite slab case, it is the escape probability. Recent generalizations of the Sobolev approach (Castor, Hummer, Kalkofen, Magnan, Rybicki) have attempted to determine the size of the necessary velocity gradient in terms of the gradients of other properties of the medium and the destruction scale (which, if destruction occurs by collisional de-excitation alone, is the same as the thermalization length). The outstanding problem seems to lie in determining the effect of the moving atmosphere on the value of the source function, rather than on the opacities, which then determine which parts of the source function distribution give rise to the observed radiation field. Results to date indicate that the moving atmosphere does not have a very large effect on the source function itself. So far, these methods have been applied mainly to the interpretation of P Cygni type profiles in terms of mass loss.

An extreme approach to determining the effect of a moving atmosphere on the source function has been developed by Lucy. He treats the scattering term alone, neglecting those contributions to the source and sink terms that come from the properties of the atmosphere alone. The value of such an approach for diagnosing the physical properties of the atmosphere remains to be demonstrated.

In a novel and controversial application of the Sobolev approach (Pecker-Gordon), the spectra of supernovae are interpreted in terms of detailed atmospheric processes. This is particularly interesting because of the variety in the treatment of supernovae from other aspects.

The extent to which wholly photospheric models can explain anomalous features in hot stars is still being explored. Following Parker's demonstration that all stars must have stellar winds, Cassinelli and Castor attempt to determine the size of wind that would result from radiative heating alone. Assuming a gray continuous absorptive opacity and LTE, they find that the ratio of the force due to radiation pressure must exceed about nine tenths that due to gravity in order to produce 
winds that are observationally significant. As an estimate of the rate of mass loss, they use the ratio $L / c v_{\infty}$ (where $L$ is the luminosity and $v_{\infty}$ the velocity at infinity), which exceeds by $c / v_{\infty}$ the limit set by Lucy and Solomon. We note, however, that this should be an upper limit, corresponding to an optically thick wind, rather than to the optically thin case treated. Attempts (Castor, Van Blerkom) to interpret Wolf-Rayet spectra in terms of purely radiative photospheres have succeeded in one case, although it is not clear at the moment that they can reproduce the high ionization level (O vI) observed in some Wolf-Rayet stars. Such work is important in establishing the real anomalies between correct photospheric models and observations.

\section{The temperature minimum}

The question of a temperature minimum, beyond which $T_{\mathrm{e}}$ increases outwards, did not arise in the classical model where $T_{\mathrm{e}}$ decreased monotonically to the boundary temperature. The dissipation of mechanical energy will cause $T_{\mathrm{e}}$ to increase outwards in a chromosphere; non-LTE effects alone may cause $T_{\mathrm{e}}$ to rise even in a radiatively controlled photosphere. This possibility was first discussed by Cayrel for $\mathrm{H}^{-}$and has since been generalized by calculations of Auer and Mihalas and of Gebbie and Thomas. Whether an outward rise in $T_{\mathrm{e}}$ actually occurs in the absence of mechanical heating will depend on the competing effects of impurity cooling and the heating by additional continua. Because the number of contributors to the continuous opacity differs from one part of the atmosphere to another and from one spectral type to another, the problem remains to be explored in detail. Observationally, it is complicated by the presence of chromospheric heating and inhomogeneities.

\section{d. Emission lines}

Here the primary problem lies in distinguishing those lines that can be explained by a wholly photospheric model from those that imply the existence of a region with quite different physical characteristics. For example, much attention is being given to the interpretation of observed $P$ Cygni profiles. The question, as yet unresolved, is whether the ratio of emission to absorption components and the high expansion velocities inferred from the blue absorption component can be explained simultaneously in terms of an extended atmosphere with a radiation driven wind. However, attempts to explain individual emission lines without invoking chromospheres or extended atmospheres have been more successful. For example, the appearance of NiII $\lambda 4634$ has been explained (Nussbaumer, Mihalas, Hummer) by a mechanism for underpopulating the lower level of the transition, as distinct from fluorescent overpopulation of the upper level. The behavior of He II $\lambda 10124$ in Of stars, on the other hand, suggests that it is formed in a chromosphere (Lockwood and Mihalas). Apparently there is no general rule by which the presence of an emission line can be interpreted in terms of fluorescent mechanisms, extended atmospheres, chromospheres, etc. Nevertheless, it is to be hoped that some kind of classification scheme may emerge from the detailed investigations now in progress.

\section{e. Photospheric shells}

We distinguish a shell as being photospheric if its state is controlled by radiative processes rather than by dissipation of mechanical energy. Thus planetary nebulae and $\mathrm{H}$ II regions, which are dealt with extensively in the reports of other commissions, are primarily photospheric shells.

The outstanding problem here is the interpretation of the infra-red excesses observed in a variety of stars. For a discussion of the problem, we refer to the summary by Pecker. In spite of its theoretical importance, most of the progress in this field has been observational and therefore lies outside the province of this commission. The important theoretical problems remain to be tackled: Are dust shells formed from stellar material or from interstellar matter? Can the bolometric correction derived from infra-red observations, together with the flux of mass and energy from UV observations, be reconciled with the effective temperature and surface gravity hitherto assigned to a particular star? Indeed do the three parameters, effective temperature, surface gravity, and chemical composition, suffice to specify an atmospheric model? 


\section{Chromosphere and corona}

Here progress over the past three years has lain more in defining than in solving the theoretical problems. The outstanding question is the degree to which chromospheric and coronal phenomena are present in the atmosphere of all stars. Almost all theoretical approaches to this problem deal with the convective production of acoustic waves, as in the Sun. The exceptions deal with additional sources of mechanical energy, such as opacity and ionization instabilities and radiation driven acoustic waves, and their necessary consequence for the production of chromospheres and mass flux. Detailed observational work is also restricted almost entirely to the Sun, with the exception of $\mathbf{P}$ Cygni profiles and other specific emission features such as the cores of the $\mathbf{H}$ and $\mathrm{K}$ lines of $\mathrm{Ca}^{+}$and $\mathrm{Mg}^{+}$in a variety of stars. The observational stellar work does, however, provide increasing evidence for atmospheric heating and mass loss beyond that attributable to radiation alone (cf. summaries by Praderie and by Feast, observations by Morton et al., and calculations by Lucy and Solomon, Cassinelli and Castor, and Nariai on the mass loss from radiative effects alone).

Following Praderie's suggestion that the existences of a chromosphere and a mass flux are intimately linked, perhaps the most likely trend in the next few years will be toward an atmospheric model for which mass flux is a necessary condition, the corona being the region from which mass is actually lost, and the chromosphere the region in which most of the mechanical energy required to produce coronal conditions is dissipated. For this, the Sun should be extremely useful as a guide but myopic as a restriction. The evolution of thinking on Wolf-Rayet and other hot stars, with regard to chromospheres, coronae and mass flux, provides an excellent example.

\section{a. Chromospheres}

The location and physics of a chromosphere depend to some extent on whether we define it by a positive outward increase in electron temperature, $T_{e}$, or by the presence of mechanical heating. They will also depend on whether we consider the corona to begin at the top of the steep rise in $T_{\mathrm{e}}$, or at the point where $T_{\mathrm{e}}$ is maximum, or at the point where the enthalpy term associated with the stellar wind exceeds the radiative losses. These questions are not entirely semantic and are at present being heavily debated as, for example, in the proceedings of the Goddard Colloquium on stellar chromospheres.

One approach to locating the base of the chromosphere, as defined by the temperature minimum, would be to treat impurity cooling as a perturbation on the $T_{\mathrm{e}}$ gradient as determined by the continuous opacity alone. Such a temperature distribution would have one minimum where collisional ionization gives way to photoionization and a second minimum where impurity cooling enters - although the second may, according to Athay, swamp the first. The subsequent increase in $T_{\mathrm{e}}$ may then result either from mechanical heating or from the effects of radiatively controlled lines as, with decreasing density, they overcome the effect of collisions. The line blanketing problem is itself sufficiently complex that the coupling between it and the mechanical heating has generally been ignored. However, the onset of dissipation from 'trapped' waves depends critically on the location of an initial outward increase in $T_{\mathrm{e}}$ (Leibacher, Ulrich, Stein), which could result either from radiative processes or from heating by the small fraction of waves never trapped. Thereafter, of course, the mechanical dissipation amplifies. Such problems, which are likely to receive much attention in the next years, tend to blur the distinction between a chromosphere defined by an outward increase in $T_{\mathrm{c}}$ and one defined by mechanical heating.

Many questions arise in attempts to solve these problems observationally. What part of a line profile can be used, i.e., when does the source function go to the Planck function at the local value of $T_{\mathrm{e}}$ ? How is the source function affected by coherent scattering versus complete redistribution in the line wings? What is the connection between the scaling factor, $\Delta v_{\mathrm{D}}$, and microturbulence, and what is the physical significance of each? What is the effect of molecular lines? To what extent is $T_{e}$ inhomogeneous near the minimum, and what effect does this have on the diagnostics? What is the physical basis for attempts at scaling from the Sun to other stars?

Another problem equally important as that of the solar temperature minimum is the behavior of $T_{\mathrm{e}}$ between the minimum and the corona. Rocket UV studies (Avrett, Kalkofen, Noyes, Vernazza) 
have confirmed the visual eclipse results of a decade ago (Thomas, Athay, Pottasch), which indicated that $T_{\mathrm{e}}$ rises rapidly above the temperature minimum, then levels off to a more gradual rise for about the next $1000 \mathrm{~km}$, and finally increases steeply to the corona. The far-UV studies also show an additional small plateau between $10^{4} \mathrm{~K}$ and $10^{6} \mathrm{~K}$ in the steep temperature rise. This plateau, predicted by earlier work, establishes radiative cooling as a controlling process in the steep temperature rise. The agreement between the visual and far-UV studies confirms the essential correctness of the diagnostic method, which depends critically on the non-LTE factors, $b_{1}$ and $b_{2}$, for hydrogen.

These analyses also indicate the very small height of the solar chromosphere - something between $1000 \mathrm{~km}$ and $2000 \mathrm{~km}$. This result is of particular interest in relation to studies of Wolf-Rayet stars, in which the entire visual spectrum is now interpreted as being formed in a coronal region, again implying a small height for a chromosphere. The question then arises of whether we should expect all chromospheres to be of relatively small extent.

In computations of the distribution of mass accompanying a given temperature distribution, we again encounter the problems associated with the introduction of ad hoc turbulence parameters. These have been discussed in the section on line profiles; here we need stress only that the relation between the 'turbulence' used in the absorption coefficient and that used for the momentum balance is presently obscure.

The effect of any inhomogeneities in the structure of the atmosphere remains uncertain. There is some indication that for sufficiently small elements, the sum of the radiative plus mechanical fluxes is constant (Evans, Catano, Thomas). Observationally, it seems clear that for elements as large as sunspots, this assumption would not be valid. The extent to which such theories of flux conservation are valid is particularly important with regard to the variety of line profiles observed over small regions and plages. Stellar counterparts exist in, for example, the spectra of Am and Ap stars, for which the interpretations concentrate more on the thermodynamic effect of inhomogeneities than on the microscopic response of parameters involved in line formation.

\section{b. Coronas}

Coronal models in which mechanical energy is balanced against radiative loss have been computed for a variety of stars with solar-type sources of mechanical energy. The results are characteristic of models based on this limited approach.

Reference has already been made in this report to two aspects of coronal structure that have received particular attention over the last three years. The first is the small extent of chromospheres relative to coronas; the second is the realization that the primary characteristic of the corona is its mass flux.

Over the past twenty years, estimates of the height at which the corona begins have decreased steadily from some $50000 \mathrm{~km}$ above the visual eclipse limb (Aller's textbook) to some $5000 \mathrm{~km}$ (Woolley and Allen), down to the present estimate of some $2000 \mathrm{~km}$. These results indicate that the mechanical energy available to raise $T_{\mathrm{e}}$ above its radiative-equilibrium value is stored for some short distance above the base of the photosphere and then dissipated over a small height range. A small fraction of the original mechanical energy passes through the chromosphere to be dumped in the corona, where it serves to blow off everything above that level.

When it was first recognized that the corona was hot, its basic character was thought to be determined by the balance between mechanical dissipation of energy and radiative loss. Later it was believed that the mechanical energy dissipated was balanced by thermal conduction downward to the chromosphere and outward to the extended corona. Then Parker's work on stellar winds laid the basis for the current belief that the state of the corona is fixed mainly by the wind it produces.

\section{PART B : BIBLIOGRAPHY}

\section{Subphotosphere}

Castellani, V., Renzini, A. 1969, 'Physical Conditions in the Convective Envelopes of Stars', Ap. Space Sci., 3, 283.

Érgma, E. $\quad 1971$, 'Density Inversion in the Convective Envelopes of Stars', Sov. Astr. - AJ, 15, No. 1. 
Grinin, V. P. $\quad 1972$, Astrofizika, 8, No. 1.

Ivanov, L. N. $\quad 1971$, Astrofizika, 7, No. 1.

Mizuno, S., Mishida, M. 1969, 'On the Structure of the Outer Solar Convection Zone', Publ. Astr. Soc. Japan, 21, No. 2.

Petrukhin, N. S. 1970 , Soln. Dann., No. 3.

Schatzman, E. 1969, 'Gravitational Separation of the Elements and Turbulent Transport', Astr. Ap., 3, 331.

Stickland, D. J., Whelan, J. A. J. $\quad$ 1972, 'Short Comm. On the Origin of the Am Phenomenon', M.N.R.A.S., 155, 11P.

Auré, J.-L. 1971, 'Overstable Damping in a Stellar Semi-Convective Zone', Astr. Ap., 11, 345.

Bhatia, P. K. 1971, 'On Thermal-Convective Instability in a Stellar Atmosphere', Publ. Astr. Soc. Japan, 23, 181.

Böhm-Vitense, E. $\quad$ 1970, 'Convection in A and F Stars and Metallicism', Astr. Ap., 8, 299.

Böhm-Vitense, E. 1971, 'Convection and Metal Abundance', Astr. Ap., 14, 390.

Busse, F. H. $\quad 1970$, 'Differential Rolation in Stellar Convection Zones', $A p . J ., 159,629$.

Defouw, R. J. $\quad 1970$, 'Thermal-Convective Instability', $A p . J ., 160,659$.

Durney, B. $\quad$ 1970, 'Nonaxisymmetric Convection in a Rotating Spherical Shell', Ap. J., 161, 1115.

Gabriel, M. 1970, 'On the Mechanism of Formation of Semi-Convective Zone in Stars', Astr. Ap., 6, 124.

Mullan, D. J. 1971, 'Cellular Convection in Model Stellar Envelopes', M.N.R.A.S., 154, 467.

Ponomarenko, Yu. B. 1969, 'Generation of Stellar Magnetic Fields by Convective Motions in the Surface Layers', Sov, Astr. - AJ, 13, No. 2.

Souffrin, P. 1970, 'Hydrodynamics of an Atmosphere Excited by an Underlying Turbulent Convective Zone', Astr. Ap., 7, 227.

Unno, W. 1969, 'Theoretical Studies on Stellar Stability II. Undisturbed Convective Nongrey Atmospheres', Publ. Astr. Soc. Japan, 21, No. 3.

Vardya, M. S. 1970, 'On Density Inversion in the Convective Zones of Stars', Observatory, 90, 69.

Watson, W. D. 1971, 'Particle Diffusion in Stars Having Shallow Convective Envelopes', Astr. Ap., 13, 263.

\section{Collisionally controlled photosphere}

a. Colors, models - quasi-empirical

Bahng, J. $\quad$ 1969, 'Infra-red Colours of G, K and M Stars', M.N.R.A.S., 143, 73.

Baluteau, J. P. $\quad$ 1971, 'Solar Brightness Measurement Between 12 and 24 Microns', Astr. Ap., 14, 428.

Battistini, P. L., Fracassini, M., Pasinetti, L. E. 1971, 'Variation of the Gradient and Balmer Discontinuity During the Eclipse of the Variable RW Tauri: Comparison Between Observations and Theory', Ap. Space Sci., 14, 438.

Bell, R. A. $\quad 1970$, 'The Calibration of Narrow Band Photometry I', M.N.R.A.S., 148, 25.

Bell, R. A. $\quad 1970$, 'Interpretation of the Colour-Colour Diagram of M92', M.N.R.A.S., 149, 179.

Bell, R. A. $\quad$ 1971, 'Theoretical Colours for F and G Dwarf Stars', M.N.R.A.S., 154, 343.

Bell, R. A. $\quad 1971$, 'The Temperatures, Abundances and Gravities of F Dwarf Stars', M.N.R.A.S., 155, 65.

Bell, R. A. 1972, 'Short Comm. The Influence of Lines of Various Metals on the Solar Colours', M.N.R.A.S., 156, 13P.

Bell, R. A., Gottliev, D. M. 1971, 'The Calibration of Narrow-Band Photometry-II. The Gravity and Doppler-Broadening Velocity of $\mathrm{G}$ and $\mathrm{K}$ Giants', M.N.R.A.S., 151, 449.

Bell, R. A., Rodgers, A. W. 1969 , 'The Influence of Line Blanketing Upon the Colours of Cepheids and F-K Supergiants', M.N.R.A.S., 142, 161.

Bolton, C. T. 1971, 'Spectral Synthesis of Low-Dispersion Luminosity Criteria in A and F Type Stars', Astr. Ap., 14, 233.

Breger, M., Kuhi, L. V. $\quad 1970$, 'Effective Temperatures, Gravities, and the Mass Determination of A and F Stars', Ap. J., 160.

Brown, R. H., Davis, F., Herbison-Evans, D., Allen, L. R. $\quad$ 1970, 'A Study of $\gamma^{2}$ Velorum With a Stellar Intensity Interferometer', M.N.R.A.S., 148, 103.

Carruthers, G. R. 1971, 'Far-Ultraviolet Spectra and Photometry of Perseus Stars', Ap. J., 166, 349.

Davis, F., Morton, D. C., Allen, L. R., Brown, R. H. 1970, 'The Angular Diameter and Effective Temperature of Zeta Puppis', M.N.R.A.S., 150, 45.

Davis, J., Webb, R. J. 1970, 'Ultraviolet Fluxes and Bolometric Corrections for Late B to F MainSequence Stars', Ap. J. 159. 
Dickens, R. J., Penny, A. J. 1971, 'Effective Temperatures and Gravities for A- and F-Type Stars in the Delta Scuti Region', M.N.R.A.S., 153, 287.

Gay, J. 1970, 'Interprétation des mesures spectrophotométriques solaires en infrarouge lointain', Astr. Ap., 7, 24.

Gustafsson, B. 1971, 'A Feautrier-Type Method for Model Atmospheres Including Convection', Astr. Ap. $10,187$.

Hearnshaw, J. B. 1972, 'The Effect of Microturbulence of UBV Colours', Observatory, 92, 43.

Herbison-Evans, D., Brown, R. H., Davis, J., Allen, L. R. 1971, 'A Study of $\alpha$ Virginis with an Intensity Interferometer', M.N.R.A.S., 151, 161.

Holweger, H. 1970, 'Depression of the Solar Continuous Spectrum by Line Absorption and the Balmer Continuum', Astr. Ap., 4, 11.

Hunger, K., Klinglesmith, D. $\quad 1969$, 'The Atmosphere of the Helium-Carbon Star BD+10²179', Ap. J., 157.

Kemp, J. C., Swedlund, J. B., Landstreet, J. D., Angel, J. R. P. 'Discovery of Circularly Polarized Light from a White Dwarf', submitted to Comm. 36 files.

Klinglesmith, D. A. III 1972, 'An Abundance Analysis of the Photometric Standard 29 Piscium', Ap. J., 171, 79.

Klinglesmith, D. A., Hunger, K., Bless, R. C., Millis, R. L. $\quad$ 1970, 'The Atmosphere of the HydrogenDeficient Star Sigma Orionis E', Ap. J., 159.

Khokhlova, V. L. 1972, 'A Study of the Spectrum-Variable Silicon Ap Star 56 Ari', Sov. Astr. - AJ, 16 224.

Khokhlova, V. L. 1971, 'The Oblique-Rotator Model for the Brightness and Color Variability of Magnetic Ap Stars', Sov. Astr. - AJ, 15, 419.

Kodaira, K. $\quad 1970$, 'The Continuous Radiation of Bright B3 V Stars', Ap. J., 159.

Koutchmy, S., Peyturaux, R. 1970, 'Etude de continuum solaire dans l'infrarouge moyen: $\lambda=3,5 \mu$ à $\lambda=25 \mu$, Astr. Ap., 5, 470 .

Léna, P. 1970, 'Le rayonnement continu infrarouge de la photosphère solaire', Astr. Ap., 4, 202.

Mégessier, C. 1971, 'Étude d'Étoiles Ap Si 4200 Températures effectives et gravités', Astr. Ap., 10, 332.

Morton, D. C. $\quad 1969$, 'The Effective Temperatures of the O Stars', Ap. J., 158.

Morton, D. C. $\quad 1970$, 'The Effective Temperatures of Six Wolf-Rayet Stars', Ap. J., 160.

Parsons, S. B. 1971, 'Effective Temperatures, Intrinsic Colours, and Surface Gravities of Yellow Supergiants and Cepheids', M.N.R.A.S., 152, 121.

Parsons, S. B., Bouw, G. D. 1971, 'Radii, Luminosities, and Masses from Six-Colour Fitting of Galactic Cepheids and Other Supergiants', M.N.R.A.S., 152, 133.

Peterson, D. M., Scholz, M. 1971, 'Investigation of Six O-Type Spectra', Ap. J., 163, 51.

Richter, D. 1971, 'Quantitative Analysis of the O-Subdwarf HD 49798', Astr. Ap., 14, 415.

Ringuelet, A. E. $\quad 1969$, 'The Paschen Discontinuity in B Stars', Ap. Space Sci., 5, 459.

Schild, R., Peterson, D. M., Oke, J. B. 1971, 'Effective Temperatures of B- and A-Type Stars', Ap. J., $166,95$.

Schmidt, E. G. $\quad 1972$, 'The Temperature Scale of F and G Stars II. Continuum Photometry', Ap. J., 174, 605.

Schmitt, A., Scholz, M., Traving, G. 1971, 'On the Interpretation of Photometric and MKK Spectral Types of Early, Main-Sequence Stars', Astr. Ap., 12, 36.

Stalio, R. $\quad 1971$, 'Temperature Scale for B-Type Supergiants', 3rd Trieste Coll. on Supergiant Stars, Ed. M. Hack, p. 28.

Stickland, D. J. 1971, 'On the Energy Distributions of Main Sequence Stars', M.N.R.A.S., 153, 501.

Tomley, L. 1970, 'The Atmospheres of the Hot Subdwarfs HD 127493, HD 128220, and HD 113001' Ap. J., 162, 239.

Underhill, A. B. 'A Study of B6 Stars', Goddart Space Flight Center, No. X 670-72-26.

Wolf, B. $\quad$ 1971, 'The Atmosphere of the AOIb Supergiant $\eta$ Leonis', Astr. Ap. 10, 383.

\section{b. Colors, models - theoretical}

Alexander, D. R., Johnson, H. R. $\quad$ 1972, 'Model Atmospheres for Cool Supergiants Stars', Ap. J., 176, 629.

Bapu, G. S. D. 1971, 'Effective Temperatures of Some Magnetic Stars', Observatory, 91, 115.

Blumenthal, G. R., Cavaliere, A., Rose, W. K., Tucker, W. H. $\quad 1972$, 'A Model for the Centaurus X-3 Phenomenon', Ap. J., 173, 213. 
Böhm, K. H., Cassinelli, J. 1971, 'Convective Envelopes and Acoustic Noise Generation in White Dwafts', Astr. Ap., 12, 21.

Böhm-Vitense, E. 1969, 'A Simple Approximation for $\mathrm{J}_{v}\left(\delta_{v}, \kappa_{v}, \tau_{v}, \mathrm{~B}_{v}\right)$, and its Application for Temperature-Correction Procedures', Ap. Space Sci., 4, 233.

Bues, I. 1970, 'The Atmospheres of Helium Rich White Dwarfs of Spectral Type DB', Astr. Ap., 7, 91.

Bues, I. 1971, 'On Helium-Rich White Dwarfs and Cooling Sequences', Observatory, 91, 221.

Cassinelli, J. P. 1971, 'Extended Model Atmospheres for the Central Stars of Planetary Nebulae', $A p$. $J ., 165,265$.

Cassinelli, J. P. 1971, 'The Continuous Energy Distribution from Stars with Hot Extended Atmospheres', Ap. J. Letters, 8, 105.

Hummer, D. G., Mihalas, D. 1970, 'Model Atmospheres for the Central Stars of Planetary Nebulae', M.N.R.A.S., 147, 339.

Karp, A. H. 1972, 'A Modification of the Avrett-Krook Temperature-Correction Procedure', Ap. J., 173, 649.

Klinglesmith, D. A. 1971, 'Hydrogen Line Blanketed Model Stellar Atmospheres', NASA-SP 3065.

Klinglesmith, D. A., Sobieski, S. $\quad$ 1970, 'Nonlinear Limb Darkening for Early-Type Stars', Ap. J., 75, 175.

Kruusmaa, A. 1970, 'Homogeneous Net of Model Atmospheres of O-B Stars', Tartu Astrophys. Obs. Teated, 27, 20.

Kruusmaa, A. 1970, 'A Review of Constructed Model Atmospheres of O-B Stars', Tartu Astrophys. Obs. Teated, $27,3$.

Maeder, A., Peytremann, E. 1970, 'Stellar Rotation: Uniformly Rotating Stars with Hydrogen-Line Blanketed Model Atmospheres: Comparison with Observations of A-Type Stars', Astr. Ap., 7, 120.

Margrave, T. E. Jr. $\quad 1969$, 'Limb-Darkening Tables for $5040 \mathrm{~K} \leq \mathrm{Teff} \leq 7200 \mathrm{~K}$ ', P.A.S.P., 81, 116.

Napier, W. McD. 1970, 'A Method for the Construction of Model Stellar Atmospheres', M.N.R.A.S. $147,287$.

Natta, A., Priete-Martinez, A. 1970, 'Stellar Atmospheres Models of K-Giant Stars: The Influence of Opacity Sources', Ap. Space Sci., 9, 440.

Parsons, S. B. $\quad 1971$, 'Limb Darkening for Yellow Supergiants', Ap. J., 164, 355.

Peytremann, E. $\quad 1970$, 'Models of Stellar Atmospheres', Thesis Obser. de Genève.

Preite-Martinez, A., Natta, A. 1971, 'Determination of the Physical Parameters of a Stellar Atmosphere: A Method', Mem. S. A. It., 42, 561 .

Preston, G. W. 1971, 'Surface Characteristics of the Magnetic Stars', P.A.S.P., 83.

Sapar, A. 'New Flux Iteration Algorithms for Model Stellar Atmospheres', Publ. Tartu Astrophys. Obs., 40, (in press).

Sapar, A., Kruusmaa, A. 1970, 'Methods to Calculate the Improved Temperature Distribution in NonGray Stellar Atmosphers', Publ. Tartu Astrophys. Obs., 38, 3.

Sapar, A., Kruusmaa, S. 1970, 'On the Methods to Calculate the Source Function and Eddington Moments', Publ. Tartu Astrophys. Obs., 38, 29.

Schmidt, E. G. $\quad$ 1971, 'A Photometric Study for Four Classical Cepheids', Ap. J., 165, 335.

Shipman, H. L. 1971, 'Hydrogen-Rich Models for Horizontal-Branch and Post-Horizontal-Branch Stars', Ap. J., 166, 587.

Strittmatter, P. A., Wickramasinghe, D. T. 1971, 'The Atmospheres of White Dwarfs - III. The Line Spectra', M.N.R.A.S., 152, 47.

Swamy, K. S. K. $\quad$ 1969, 'Atmospheres of Extreme Metal-Deficient Stars', Ap. Space Sci., 4, 218.

Swamy, K. S. K. 1970, 'Atmospheres of Metal-Deficient Stars: Giant Stars', Astr. Ap., 8, 375.

Swamy, K. S. K. 1970, 'Atmospheric Structure of Cool Stars with Particle Opacity', Ap. J., 162, 259.

Tarafdar, S. P., Vardya, M. S. 1972, 'On Ultraviolet Stellar Fluxes. II. Importance of CO Band Absorption and Bound-Free Absorption of $\mathrm{Al} \mathrm{I}$ in A, F, G and $\mathrm{K}$ Stars', Ap. J., 178, No.2.

Trasco, J. D. $\quad 1972$, 'Light Variations in Magnetic Stars', Ap. J., 171, 569.

Underhill, A. B. 'The Ultraviolet Flux Envelopes of Main-Sequence B Stars', Goddard Space Flight Center, No. X670-72-102.

Van Citters, G. W., Morton, D. C. 1970, 'Model Atmospheres for B-Type Stars with Blanketing by Ultraviolet Lines', Ap. J., 161, 695.

Vardya, M. S. 1970, 'On $\mathrm{H}_{2} \mathrm{O}^{-}$and Cool Stars', Observatory, 90, 30.

Vardya, M. S. $\quad 1970$, 'Atmospheres of Very Late-Type Stars', Ann. Rev. Astr. Astrophys., 8, 87.

Vardya, M. S. 1970, 'Dissociation Equilibrium and Convection in Cool Supergiants', Supergiant Stars, Ed. M. Hack, Trieste, p. 207. 
Vardya, M. S. 1971, 'Atmospheres of Very Cool Supergiants - Introductory Talk', Supergiant Stars, Ed. M. Hack, Trieste, p. 190.

Wegner, G. $\quad$ 1972, 'The Spectra and Element Abundances of Cool White Dwarfs', Ap. J., 172, 451.

Wentzel, D. G. $\quad 1970$, 'On the Instability of a Stellar Envelope Due to Radiation Pressure', Ap. J., 160, 373.

Wickramasinghe, D. T., Strittmatter, P. A. 1970, 'The Atmospheres of White Dwarfs - II The Effects of Convection', M.N.R.A.S., 150, 435.

c. Abundances, integrated line intensities - quasi-empirical

Aller, M. F. $\quad$ 1970, 'A Model Atmosphere Analysis of the Ap Star $\kappa$ Cancri', Astr. Ap., 6, 67.

Bappu, M. K. V., Raghavan, N. 1969, 'An Analysis of the Cepheid Variable RT Aurigae', M.N.R.A.S., 142, 295.

Barker, T., Baumgart, L. D., Butler, D., Cudworth, K. M., Kemper, E., Kraft, R. P., Lorre, J., Rao, N. K., Reagan, G. H., Soderblom, D. R. 1971, 'Abundance Analyses of Population II Variable Stars I. W Virginis', Ap. J., 165, 67.

Baschek, B., Sargent, W. L. W., Searle, L. 1972, 'The Chemical Composition of the B-Type Subdwarf HD 4539', Ap. J., 173, 611.

Blanc-Vaziaga, M. J., Cayrel, G., Cayrel, R. 1973, 'Contribution to the Study of Supermetallicity in Late Type Giants', $A p$. $J$., (in press).

Boesgaard, A. M. $\quad 1970$, 'Lithium in Heavy-Metal Red Giants', Ap. J., 161, 1003.

Boesgaard, A. M. $\quad 1970$, 'The Ratio of Titanium to Zirconium in Late-Type Stars', Ap. J., 161, 163.

Boesgaard, A. M. 1971, 'The Lithium Content of Capella', Ap. J., 167, 511.

Branch, D., Bell, R. A. 1971, 'The Colours and Chemical Composition of the G Dwarf $\mathrm{Hr} 72$ ', M.N.R.A.S., 153, 57.

Branch, D., Bell, R. A. 1971 'An Abundance Analysis of the Ultra-Violet Deficient Subgiant HR244,' M.N.R.A.S.. 151, 289.

Cayrel, G. 1972, 'Chemical Composition of the Alleged SMR Stars', Coll. No. 17 IAU, Stellar Ages, Paris, Sept. 15-22.

Chaffee, F. H. Jr., Carbon, D. F., Strom, S. E. $\quad$ 1971, 'Abundances in Open Clusters: Model-Atmosphere Abundance Analysis of Stars in the Pleiades and Hyades Clusters', Ap. J., 166, 593.

Cohen, J. G. 1970 , 'The Spectrum of $a^{2}$ CVn. II', Ap. J., 159.

Conti, P. S. $\quad 1970$, 'Coarse Analysis of the Peculiar A Star 46 DRA', Astr. Ap., 7, 213.

Conti, P. S., Loonen, J. P. $\quad 1970$, 'Coarse Analysis of the Helium Weak B Star Iota Ori B', Astr. Ap., 8, 197.

Danziger, I. J., Jura, M. A. $\quad 1970$, 'The Hale B Star HD 137569', Ap. J., 161, 997.

De Strobel, G. C., Chauve-Godard, J., Hernandez, G., Vaziaga, M. J. 1970, 'Sodium in Late Type Stars', Astr. Ap., 7, 408.

Dickens, R. J., Franch, V. A., Owst, P. W., Penny, A. J., Powell, A. L. T. 1971, 'An Abundance Analysis of the Delta Scuti Variable 20 CVn', M.N.R.A.S., 153, 1.

Durrant, C. J. 1970, 'Rotation and Chemical Abundances in the Peculiar A Stars - I', M.N.R.A.S., $147,59$.

Evans, J. C., Schroeder, L. W. 1972, 'The Effect of Systematic $g f$-Value Errors on Stellar Curves of Growth', P.A.S.P., 84.

Faraggiana, R., van't Veer-Menneret, C. 1970, 'Analyse de l'étoile à raies metalliques 15 Vul parla methode differentielle des courbes de croissance', Compte Rendus, 270, 765.

Faraggiana, R., van't Veer-Menneret, C. 1971, 'Detailed Analysis of the Metallic-line Star 15 Vulpeculae', Astr. Ap., 12, 258.

Foy, R. $\quad$ 1972, 'Spectroscopic Study of o Per', Astr. Ap., 16, 108.

Foy, R. 1972, 'On the Solar Curve of Growth of Iron', Astr. Ap., 18, 26.

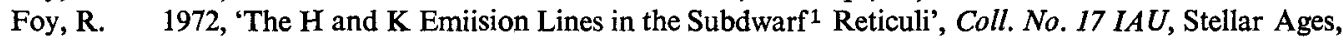
Paris, Sept. 15-22.

Gehlich, U. K. $\quad$ 1969, 'Differential Fine Analysis Sirius Versus Vega', Astr. Ap., 3, 169.

Glagolevskii, Yu. V., Leushin, V. V., Kozlova, K. I., Chunakova, N. M. 1972, 'Temperature Scale for Peculiar and Metal-Line Stars', Sov. Astr. - A.J, 15, No. 5.

Gökkaya, N. 1970, 'Quantitative Analysis and Spectral Variation of the Ap Star HD 151199', Ap. Space Sci., 6, 141.

Goon, G., Auman, J. R. $\quad$ 1970, 'Molecular Abundances in K and M Stars', Ap. J., 161, 533.

Greene, T. F. $\quad$ 1970, 'Analysis of the CO Bands in Arcturus', Ap. J., 161, 365. 
Griffin, R. $\quad 1969$, 'The Atmosphere of $\alpha$ Serpentis - I', M.N.R.A.S., 143, 223.

Griffin, R. 1969, 'Differential Curve-of-Growth Analyses of Four Late-Type Giant Stars', M.N.R.A.S., $143,381$.

Griffin, R. 1971, 'Curve-of-Growth Analysis of the Spectrum of Procyon', M.N.R.A.S., 155, 139.

Gustafsson, B., Nissen, P. E. 1972, 'The Metal-to-Hydrogen Ratio in F1 - F5 Stars, As Determined by a Model-Atmosphere Analysis of Photoelectric Observations of a Group of Weak Metal Lines', Astr. Ap., 19, 261.

Hearnshaw, J. B. 1971, 'The Old Disk Metal-Rich Subgiant 31 Aquilae', Ap. J., 168, 109.

Hirai, M. 1969, 'Spectral Analysis of a Peculiar Carbon Star, WZ Cassiopeiae', Astr. Soc. Japan, 21, No. 1.

Khokhlova, V. L. 1970, 'Inhomogeneities of Chemical Composition and Physical Conditions at the Surface of the Si II Ap Stars CU Vir and 56 Ari', Sov. Astr. - AJ, 14, No. 1.

Kodaira, K., Greenstein, J. L., Oke, J. B. $\quad$ 1970, 'The Unusual Composition of +39²4926', Ap. J., 159.

Kodaira, K., Scholz, M. 1970 , 'Spectroscopic Investigation of B3V Stars: $\imath$ Herculis $\eta$ Hydrae, HD 58343', Astr. Ap. 6, 93.

Kodaira, K., Tanaka, K. 1972, 'Oxygen Abundances of Three Population II Horizontal-Branch Stars', Publ. Astr. Soc. Japan, 24, 355.

Maehara, H. 1971, 'Spectral Analyses of Some Mira-Type Long-Period Variable Stars', Publ. Astr. Soc. Japan, 23, 313.

Molnar, M. R. 1972, 'The Helium-Weak Stars', Ap. J., 175, 453.

Nissen, P. E. 1970, 'The Metal-to-Hydrogen Ratio for Stars in the Hyades and Coma Open Clusters', Astr. Ap., 8, 476.

Orlov, M. Ya., Rodriguez, M. H., Shavrina, A. V. 1970, 'Chemical Composition of the Atmospheres of Type M Supergiants', Sov. Astr. - AJ, 14, No. 4.

Pasinetti, L. 1972, 'On the Chemical Composition and Ages of the High Velocity Giants HD 6497', Coll. No. 17 IAU, Stellar Ages, Paris, Sept. 15-22.

Peters, G. J., Aller, L. H. 1970. 'The Chemical Composition of Iota Herculis', Ap. J., 159.

Powell, A. L. T. 1970, 'The Chemical Composition of Twelve Late F. Dwarfs', M.N.R.A.S., 148, 477.

Przybylski, A. 1969, 'The Analysis of the Low Gravity Halo Star HD 214539', M.N.R.A.S., 146, 71.

Przybylski, A. 1971, 'The Analysis of the Spectrum of the Large Magellanic Cloud Supergiant HD 32034 M.N.R.A.S., 152, 197.

Przybylski, A. 1971, 'Analysis of the Spectrum of the Ao III Halo Star HD 106304', M.N.R.A.S., 153, 111.

Querci, M., Querci, F. $\quad 1970,{ }^{~} \mathrm{~N}^{14} / \mathrm{C}^{12}, \mathrm{C}^{12} / \mathrm{C}^{13}$ and $\mathrm{N}^{14} / \mathrm{N}^{15}$ in the Carbon Star UU Aur', Astr. Ap., 9, 1.

Reimers, D. $\quad 1969$, 'Quantitative Analyse des $\delta$ Scuti-Veranderlichen $\delta$ Delphini', Astr. Ap. 3, 94.

Rice, J. B. 1970, 'An Oblique Rotator Model for the Magnetic and Spectrum Variable HD 173650', Astr. Ap., 9, 189.

Rodgers, A. W. $\quad 1969$, 'The Composition and Age of $\delta$ Pavonis', M.N.R.A.S., 145, 151.

Rogerson, J. B. Jr. $\quad 1969$, 'On the Abundance of Iron in the Solar Photosphere', Ap. J., 158.

Ross, J. E., Aller, L. H. $\quad$ 1970, 'The Manganese Star Iota Coronae Borealis', Ap. J., 161, 189.

Schmidt, E. G. $\quad$ 1971, 'An Analysis of the Iron Spectrum of Four Cepheids', Ap. J., 170, 109.

Seligman, C. E., Aller, L. H. $\quad 1970$, 'A Model Atmosphere Analysis of the Binary 112 Herculis', Ap. Space Sci., 9, 461.

Sievers, H. C. 1969 , 'An Abundance Analysis of the B3 Star HD 37058', P.A.S.P., 81, 33.

Smith, M. A. 1971, 'Microturbulence and Abundances in Am Stars: Inferences Concerning Metallicism', Astr. Ap., 11, 325.

Spinrad, H., Luebke, W. R. Jr. 1970, 'A Curve-of-Growth Analysis of the Super-Metal-Rich G Dwarf HR 72', Ap. J., 160.

Spite, M., Spite, F. $\quad 1973$, 'Study of the Abundances of Heavy Elements in F-G Type Stars I - Differential Analysis of Two Metal Deficient Stars: HR 646 and HR 860', Astr. Ap., (in press).

Spite, M. $\quad 1973$, 'The Helium Content of 99 Her', Ap. J. Letters, (in press).

Stalio, R. 1971, 'Preliminary Study on Early-Type Supergiants in $\mathrm{h}$ and $\chi$ Persei', 3rd Trieste Coll. on Supergiant Stars, Ed. M. Hack, p. 85.

Strohback, P. 1970, 'Atmosphärensiruktur und chemische Zusammensetzung einiger K-Hauptreihensterne', Astr. Ap., 6, 385.

Strom, S. E., Strom, K. M., Carbon, D. F. 1971, 'A Model-Atmosphere Analysis of Five Apparently Super-Metal-Rich K Giants', Astr. Ap., 12, 177.

Thomas, M. 1971, 'Étude de la déficience en métaux dans l'atmosphère d'une étoile F: 15 Pegasi', Thesis (3ème cycle, Paris VII University, France). 
Tomkin, J. 1972, 'The Composition of the Subdwarf Groombridge 1830', M.N.R.A.S., 156, 349.

Tomley, L. J., Wallerstein, G., Wolff, S. C. 1970, 'A Spectroscopic Analysis of the Silicon Star HD 34452', Astr. Ap., 9, 380.

Utsumi, K. 1970, 'Abundance Analysis of Cool Carbon Stars', Publ. Astr. Soc. Japan, 22, 93.

van Paradijs, J. A. $\quad 1971$, 'A Curve of Growth Analysis of $\delta$ Cep', Astr. Ap., 11, 299.

Vilhu, O. $\quad$ 1972, 'Spectroscopic Analysis of the Weak-Helium-Line Star $\alpha$ Sculptoris', Ann. Acad. Scientiarum Fennicae, Series A, VI, Physica, 394.

Viotti, R., Ricciardi, O. $\quad 1971$, 'On the Spectral Analysis of the Superluminous Emission Line Stars', 3rd Trieste Coll. on Supergiant Stars.

Watson, R. D. 1971, 'Atmospheric Abundances in the Beta Cephei Stars', Ap. J., 169, 343.

Wegner, G. $\quad$ 1972, 'The Spectra and Element Abundances of Cool White Dwarfs', Ap. J., 172, 451.

Williams, P. M. 1971, 'Stellar Compositions From Narrow-Band Photometry - I', M.N.R.A.S., 153, 171.

Williams, P. M. 1972, 'Short Comm. The Spectrum of Omicron Virginis', M.N.R.A.S., 115.

Wyckoff, S. $\quad$ 1970, 'Near-Infrared Spectra of M, S, and Carbon Stars', Ap. J., 162, 203.

Zappala, R. R. 1972, 'Lithium Abundances of Stars in Open Clusters', Ap. J., 172, 57.

Zielke, G. 1970, 'Spektroskopische Analyse von fünf Sternen der $\gamma$ Leonis-Gruppe', Astr. Ap., 6, 206.

\section{d. Abundances, integrated line intensities - theoretical}

Gussmann, E. A. 1970, 'Weighting Functions and nonLTE', Observatory, 90, 260.

Guthrie, B. N. G. 1970, 'Light-Element Abundances of Peculiar A Stars', Ap. Space Sci., 8, 172.

Havnes, O., Conti, P. S. $\quad$ 1971, 'Magnetic Accretion Processes in Peculiar A Stars', Astr. Ap., 14, 1.

Michaud, G. $\quad 1970$, 'Diffusion Processes in Peculiar A Stars', Ap. J., 160.

Michaud, G., Vauclair, S. $\quad 1972$, 'Diffusion and the 'He Abundance in 3 cen A', Ap. J. Letters, 11, 117.

Rosendhal, J. D. 1969, 'The Effect of Electron Scattering on Curves of Growth', Ap. Space Sci., 4, 419.

Ruhm, H. 1969, 'Contribution Functions' Astr. Ap., 3, 277.

Thomas, R. N., Pecker, J.-C. $\quad$ 1970, 'Saturation in Fraunhofer Lines', Observatory, 90, 207.

Tuominen, I. V., Vilhu, O. $\quad$ 1970, 'Abundances and Stellar Rotation', Ap. J. Letters, 6, 143.

Tuominen, I. V., Vilhu, O. 1970, 'On the Effect of Rotation on Stellar Absorption Lines', Stellar Rotation, Ed. A. Slettebak, p.110.

Vauclair, S. $\quad$ 1972, 'Lithium as a Stellar Age Indicator', Coll. No. 17 IAU, Stellar Ages, Paris, Sept. 15-22.

Vauclair, S., Reeves, H. 1972, 'Spallatian Processes in Stellar Surfaces: Anamalous Helium Ratios', Astr. Ap., 18, 215.

Vilhu, O., Tuominen, I. V. 1971, 'The Effect of Uniform Stellar Rotation on Absorption Line Strengths and Abundances', Astr. Ap., 13, 136.

e. Line profiles, line effects, including blanketing - quasi-empirical

Badalyan, O. G., Livshits, M. A. 1971, 'Profiles of Lines in the Active and Undisturbed Photosphere', Sov. Astr. - AJ, 15, No. 1.

Barcza, S. $\quad$ 1972, 'Highly Excited Hydrogen Lines in Stellar Spectra. II', Ap. Space Sci. 16, 372.

Boyer, R. $\quad 1970$, 'Expérimentations numériques sur des profils de raies photosphériques', Astr. Ap., 8, 134.

Gompertz, G., Hindmarsh, W. R. 1969, 'The Interpretation of the Wing of the Solar Line Ho', M.N.R.A.S., 142, 97.

Leckrone, D. S. 1971, 'The He I Line Profiles in Normal B Type Spectra', Astr. Ap., 11, 387.

Pustylnik, I. 1971, 'On the Determination of the Rotational Velocities of Non spherical Stars', Tartu Astr. Obs. Teated, 35, 51.

Schmidt, E. G. $\quad 1970$, ' $\mathrm{H} \alpha$ as a Temperature Indicator in Cepheids', Ap. J., 162, 871.

Smith, A. M. 1972, 'Rocket Spectroscopy of Zeta Orionis', Ap. J., 172, 129.

Smith, A. M. $\quad 1970$, 'Rocket Spectroscopy of Zeta Puppis', Ap. J., 160.

f. Line profiles, line effects, including blanketing - theoretical

Bell, R. A. 1970, 'The Nitrogen Abundance of $v$ Indi', M.N.R.A.S., 150, 15.

Kuusik, I. 1971, 'The Dispersion Formula for Broadening of Hydrogen Lines for Early-Type Stars', Publ. Tartu Astrophys. Obs., 39, 68.

Natta, A., Preite-Martinez, A. 1971, 'On the Role of the Collisionally Broadened Wings of Strong Lines in the Blanketing Problem', Ap. Space Sci., 13, 148.

Sapar, A., Kuusik, I. 1973, 'On Line Profile Function for Hydrogenic Atoms', Publ. Tartu Astrophys. Obs., 40, (in press). 
Wickramasinghe, D. T., Strittmatter, P. A. 1970, 'Rotation and the DC White Dwarfs', M.N.R.S.A., $147,123$.

Yamashita, Y. 1970, 'A Remark on the Determination of Electron Presure from the Analysis of Stellar Spectra', Publ. Astr. Soc. Japan, 22, 239.

\section{g. Inhomogeneities - quasi-empirical}

Norris, J., Baschek, B. 1973, 'A model for the Helium Spectrum Variable $a$ Centauri', submitted to Comm. 36 files.

Sotirovski, P. $\quad$ 1971, 'The Molecular Spectrum of Sunspot Umbrae', Astr. Ap., 14, 319.

Smith, M. A. 1972, 'Metallicism in Border Regions of the Am Domain I. Extremely Young Am Stars in the Orion 1c Association', Ap. J., 175, 765.

Teplitskaya, R. B., Turchina, V. D. $\quad 1969$, 'Equivalent Line Widths in Sunspots', Sov. Astr. - AJ, 13, No. 1 .

Vardya, M. S. $\quad 1970$, 'Stellar Spots and 73 Draconis', Observatory, 90, 155.

\section{h. Inhomogeneities - theoretical}

Dicke, R. H. $\quad$ 1970, 'Why are Sunspots Dark and Faculae Bright?', Ap. J., 159.

Howard, R. 1971, 'Solar Magnetic Fields - Large Scale', P.A.S.P., 83.

Khlystov, A. I., Sitnik, G. F. $\quad 1970$, 'Physical Conditions in Faculae and the Photosphere at the Level of Formation of the CH Molecule', Sov. Astr. - AJ, 14, No. 6.

Kunkel, W. E. $\quad 1970$, 'On the Spectra of Stellar Flares', Ap. J., 161, 503.

Ponomarenko, Yu. B. 1970, 'The Formation Mechanism of Active Regions', Sov. Astr. - AJ, 14, No. 1.

Soru-Escaut, I., Semel, M. 1971, 'Étude de l'élargissement des raies de la série de Balmer dans le spectre d'une éruption', Astr. Ap., 12, 340.

Talwar, S. P., Singla, M. $\quad 1972$, 'Thermal Instability with Velocity Shear', Ap. J., 172, 155.

Ulrich, R. K. 1970, 'Convective Energy Transport in Stellar Atmospheres III. Multi-Stream Atmospheres III', Ap. Space Sci., 9, 80.

Vainshtein, S. I. 1971, 'Solar Magnetic Field Generation by Cyrotropic Turbulence', Sov. Astr. - AJ, 15 , No. 3 .

Wolff, C. L. $\quad$ 1972, 'Free Oscillations of the Sun and Their Possible Stimulation by Solar Flares', Ap. J., $176,833$.

\section{i. Polarization - quasi-empirical}

Angel, J. R. P. Landstreet, J. D. 'Further Polarization Studies of Grw $+70^{\circ} 8247$ and Other White Dwarfs', Columbia Astrophys. Lab. Contribution \#26.

Débarbat, S., Dumont, S., Pecker, J. C. 1970, 'La polarisation du spectre continu au bord du disque solaire', Astr. Ap., 8, 231.

Débarbat, S., Dumont, S., Pecker, J.-C. $\quad 1970$, 'Interpretation of Optical Solar Polarization', Ap. J. Letters, 6, 251.

Dumont, S., Pecker, J.-C. 1971 , 'Influence de l'absorption dans les raies sur la polarisation du spectre continu', Astr. Ap., 10, 118.

\section{j. Polarization - theoretical}

Collins, G. W. II 1970, 'Intrinsic Polarization in Nongray Atmosphere', Ap. J., 159.

Dumont, S. 1973, 'Numerical Solution of the Transfer Equation for Polarized Radiation', J.Q.S.R.T., (in press).

Harrington, J. P. 1970, 'Polarization of Radiation from Stellar Atmospheres. The Grey Case', Ap. Space Sci., 8, 227.

Pacholczyk, A. G., Swihart, T. L. 1970, 'Polarization of Radio Sources. II. Faraday Effect in the Case of Quasi-Transverse Propagation', Ap. J., 161, 415.

k. Molecules - quasi-empirical

Faÿ, T. D., Johnson, H. R. 1972, 'A Search for $\mathrm{C}_{2}$ in Spectra of HD 201626 and the Sun', P.A.S.P., 84, 284.

Faÿ, T. D. Jr., Van Citters, G. W. 1973, 'Computed and Observed Cyanide-Radical Spectra of Two N Stars Near $6708 \AA^{\prime}, A p$. J., submitted.

Faÿ, T. D. Jr. 1971, 'Computed and Observed Cyanide-Radical Spectra of Three N Stars in the Infrared', Ap. J., 168, 99. 
Johnson, H. R., Marenin, I. 1972, 'Radiative Opacity Due to the Red System of CN', J.Q.S.R.T., 12, 189.

Khlystov, A. I. 1970, 'A Mechanism for the Formation of the CN Lines in the Solar Photosphere', Sov. Astr. - AJ, 14, No. 1.

Kozhenikov, N. I., Polonskii, V. V. 1969, 'Structure of the CO Layer in the Solar Atmosphere', Sov. Astr. - $A J, 13$, No. 2.

Profir'eva, G. A., Sitnik, G. F. 1971, 'Determination of the Vibrational Temperature in the Photosphere of the Sun from the Weak Bands of the CN Violet System', Sov. Astr. - AJ, 15, No. 1.

Sitnik, G. F., Polonskii, V. V. 1970, 'Constancy of the Temperature in the CO Molecule Layer of the Solar Atmosphere', Sov. Astr. - AJ, 14, No. 3.

\section{Molecules - theoretical}

Kuusik, I. 1970, 'The Absorption Coefficient of Quasi-Molecular Hydrogen', Publ. Tartu Astrophys. Obs., 38, 68.

Tarafdar, S. P., Vardya, M. S. 1973, 'On Ultraviolet Stellar Fluxes. II Importance of CO Band Absorption and Bound-Free Absorption of ALI in A, F, G and K Stars', submitted to Comm. 36 files.

Tarafdar, S. P., Vardya, M. S. 1973, 'On Ultraviolet Stellar Fluxes. III. Importance of $\mathrm{H}_{2}$ Lyman Band Absorption in Sun and Other Stars', submitted to Comm. 36 files.

$\mathrm{m}$. Velocities, including hydromagnetics - quasi-empirical

Aydin, C. $\quad$ 1972, 'Atmospheres of A-Type Supergiants', Astr. Ap., 19, 369.

Badalyan, O. G., Livshits, M. A. 1970, 'The Difference of the Turbulent Velocities in the Active and Unperturbed Photosphere', Sov. Astr. - AJ, 13, No. 5.

Chaffee, F. H. Jr. 1970, 'Microturbulence in Main Sequence Stars', Astr. Ap., 4, 291.

Evans, J. C., Elste, G. $\quad 1971$, 'Line Broadening in $y$ Equilei', Astr. Ap., 12, 428.

Gonczi, G., Roddier, F. $\quad$ 1971, 'A Model of the Solar Photospheric Velocity Field', Astr. Ap., 11, 28.

Hardorp, J., Scholz, M. 1971, 'The Effect of Rapid Rotation on Radiation from Stars III. Strong Helium I Lines', Astr. Ap., 13, 353.

Hutchinson, R. B. 1971, 'Turbulence Velocities in the Atmosphere of Alpha Orionis', Ap. J., 170, 551.

Maehara, H. 1971, 'A Study on Differential Radial Velocities in the Spectra of Chi Cygni and Omicron Ceti', Publ. Astr. Soc. Japan, 23, 503.

Nariai, K. $\quad 1970$, 'A Model for a Shell Star, Zeta Tauri', Publ. Astr. Soc. Japan, 22, 313.

Plaskett, H. H. $\quad$ 1970, 'Limb Darkening and Solar Rotation - II', M.N.R.A.S., 148, 149.

Rosendhal, J. D. $\quad 1970$, 'Microturbulence in A-Type Supergiants', Ap. J., 160.

Rosendhal, J. D., Wegner, G. $\quad 1970$, 'Spectrum Variations in A-Type Supergiants', Ap. J., 162, 547.

\section{n. Velocities, including hydromagnetics - theoretical}

Baglin, A. 1973, 'Short Period Variable Stars IX. Rotation and Mixing in the Outer Layers of A Stars', Astr. Ap., (in press).

Castellani, V., Puppi, L., Renzini, A. 1971, 'Physical Conditions in the Convective Envelope of Stars', Ap. Space Sci., 10, 136.

Clement, M. J. 1972, 'Meridian Circulation with Rapid Differential Rotation in Radiative Stellar Envelopes', Ap. J., 175, 135.

Davis, C. G. Jr. $\quad 1972$, 'A W Virginis Model Using Radiation Transfer', Ap. J., 172, 419.

Durney, B. R. 1972, 'The Effect of Radiative Equilibrium on the Photospheric Angular Velocity', Ap. J., $172,479$.

Fischel, D., Nethery, C. M., Sparks, W. M. 1971, 'On the Outer Layers of Model Moving Atmospheres', Ap. J., 163, 649.

Golin'ko, V. I. 1970, 'Emission from the Wake of a Shock Wave in Stellar Atmospheres', Sov. Astr. - AJ, 14, No. 1.

Gonczi, G., Roddier, F. 1971, 'A Model of the Solar Photospheric Velocity Field', Astr. Ap., 11, 28.

Gurtovenko, É. A., Ratnikova, V. A. 1971, 'Effect of Macroturbulence on Fraunhofer Line Profiles', Sov. Astr. - AJ, 14, No. 5.

Iroshnikov, R. S. 1969, 'The Nature of the Sun's Differential Rotation', Sov. Astr. - AJ, 13, No. 1.

Keller, C. F., Mutschlecner, J. P. 1970, 'Atmospheres of Classical Cepheid Variables', Ap. J., 161, 217.

Klimishin, I. A. $\quad 1972$, 'On Thermal Waves in Stars', Ap. Space Sci., 16, 432.

Kopecký, M. 1970, 'Electric Conductivity in Photospheres of Stars of Spectral Types F, G and K', Bull. Astr. Inst. Czech., 21, 231. 
Kopecký, M. 1970, 'Difference in Magnetohydro-dynamic Properties of Photospheres of Dwarf and Giant Stars of Spectral Types F, G and K', Bull. Astr. Inst. Czech., 21, 310.

Latour, J. 1970, 'Envelope Models for A Type-Stars. The Density Inversion Zone with the Mixing Length Theory', Astr. Ap., 9, 277.

Marlborough, J. M. $\quad 1970$, 'Models for the Envelopes of Be Stars. II', Ap. J., 159.

Marlborough, J. M. Roy, J.-R. 1971, 'Models for the Envelopes of Be Stars. III. Pole-on Stars', Ap. J., $169,327$.

Pustylnik, I. 'Radiative Transfer in the Atmospheres of Rotating Stars', Trans. Acad. Sci. Estonian S.S.R., 19, 428.

Sakurai, T. 1970, 'The Negative Viscosity Phenomenon Associated with the Rossby Wave on the Differentially-Rotating Solar Photosphere', Publ. Astr. Soc. Japan, 22, 177.

Swamy, K. S. K. 1969, 'Convection in Normal Stars and Metal-Deficient Stars', Ap. Space Sci., 3, 552.

Ulrich, R. K. 1970, 'Convective Energy Transport in Stellar Atmospheres II. Model Atmosphere Calculation', Ap. Space Sci., 7, 183.

Ulrich, R. K. 1970, 'The Five-Minute Oscillations on the Solar Surface', Ap. J., 162, 993.

o. Non-plane parallel, extended, and expanding atmospheres, including mass loss - quasi-empirical

Couturier, P., Leblanc, Y. $\quad$ 1970, 'On the Origin of the Solar Wind Velocity Variations', Astr. Ap., 7, 254.

Cowling, T. G. 1969, 'The Solar Wind', Observatory, 89, 217.

Gahm, G. F. $\quad 1970$, 'On the Mass Loss from T Tauri Stars', Astr. Ap., 8, 73.

Huang, S. 1970, 'Light Curve for Eclipsing Stars with Scattering Envelopes and its Application to the V444 Cygni Binary System', Ap. J., 161, 1033.

Kitchin, C. R. $\quad 1970$, 'A Method for Determining the Sizes of the Gaseous Envelopes Surrounding Be Stars', M.N.R.A.S., 150, 455.

Martini, A. $\quad 1969$, 'On the Interpretation of S Doradus', Astr. Ap., 3, 443.

Ponomarenko, Yu. B. 1972, 'Temperature and Velocity Differences for Components of the Solar Wind', Sov. Astr. - AJ, 15, No. 5.

Tsuji, T. 1971, 'Atmospheric Structure, Mass Loss, and Chemical Composition in R Andromedae and R Cygni', Publ. Astr. Soc. Japan, 23, 275.

Viotti, R. 1969, 'Investigations on the Emission Lines in Stellar Spectra', Thesis, Scuola di Perfezionamento in Fisica, Rome Univ.

Wilson, W. J., Barrett, A. H., Moran, J. M. 1970, 'OH Radio Emission Associated with Infrared Stars', Ap. J., 160.

p. Non-plane parallel, extended, and expanding atmospheres, including mass loss - theoretical

Alazraki, G., Couturier, P. 1971, 'Solar Wind Acceleration Caused by the Gradient of Alfvén Wave Pressure', Astr. Ap., 13, 380.

Bisnovatyi-Kogan, G. S., Nadyozhin, D. K. 1972, 'The Evolution of Massive Stars with Mass Loss', Ap. Space Sci., 15, 353.

Cassinelli, J. P., Castor, J. I. 1973, 'Optically Thin Stellar Winds in Early Type Stars', submitted to Comm. 36 files.

Goldreich, P., Julian, W. H. $\quad$ 1970, 'Stellar Winds', Ap. J., 160.

Henriksen, R. N., Rayburn, D. R. 1971, 'Relativistic Stellar Wind Theory: 'Near' Zone Solutions', M.N.R.A.S., 152, 323.

Hillendhal, R. W. 1970, 'Observational Confirmation of a Mechanism for the Production of Extended Stellar Atmospheres', P.A.S.P., 82, 1231.

Hutchings, J. B. $\quad 1969$, 'Expanding Atmosphere in OB Supergiants - III', M.N.R.A.S., 144, 235.

Hutchings, J. B. 1970, 'Expanding Atmospheres in OB Supergiants IV. A Mass-Loss Survey', M.N.R.A.S., 147, 161.

Hutchings, J. B. $\quad 1970$, 'Expanding Atmospheres in OB Supergiants V', M.N.R.A.S., 147, 367.

Jockers, K. 1970, 'Solar Wind Models Based on Exospheric Theory', Astr. Ap., 6, 219.

Marlborough, J. M. 1971, 'Radiation Pressure and Accelerating Stellar Winds', Ap. J., 169, 441.

Mushotzky, R. F., Solomon, P. M., Strittmatter, P. A. 1972, 'Radiation-Pressure-Driven Mass Loss from Quasi-Stellar Objects', Ap. J., 174, 7.

Swamy, K. S. K. 1972, 'Infrared Observations and Atmospheres of Cool Stars I. Support Mechanisms for Circumstellar Shell', Proc. 17th Liege Int'l. Astrophys. Symposium on Astr. Spectra in the Infrared and Microwave Regions. 
Vijk, T. $\quad$ 1970, Publ. Tartu Astrophys. Obs., 38.

Vijk, T. $\quad$ 1971, Publ. Tartu Astrophys. Obs., 39.

Wentzel, D. G. $\quad$ 1970, 'On the Instability of a Stellar Envelope Due to Radiation Pressure', $A p . J ., 160$.

\section{Radiatively controlled photosphere}

Athay, R. G. 1972, Radiation Transport in Spectral Lines, D. Reidel Publishing Company, DordrechtHolland.

Mihalas, D. 1970, Stellar Atmospheres, W. H. Freeman and Company, San Francisco.

\section{a. Colors, models - quasi-empirical}

Gros, M. 1972, 'On the Ultraviolet Spectra of Sirius', Thèse de 3e cycle, Paris University.

Heap, S. R. 'Observations of $O$ and Of Stars', Goddard Space Flight Center No. X671-71-88.

Khlistov, A. I., Sitnik, G. F. $\quad$ 1971, Astronomich. Jhur., 47, No. 6.

Maeder, A. $\quad$ 1971, 'An Observational Evidence of NonLTE Effects for O-Type Siars', Astr. Ap., $13,444$.

Parsons, S. B. 1970, 'A Fine Analysis of Two Yellow Supergiants II. The Continuous Energy Distributions', $A p . J ., 159$.

Rublev, S. V. 1972, Astrofizicheskie Issledovania (=Izvestia of the Special Ap. Observatory) 4.

Sarichev, A. P. $\quad$ 1971, Astronomich. Zhur., 47, No. 6; 48, No. 6.

Smith, M. A., Strom, S. E. 1969,'Observational Results Concerning Departures from LTE in Late B Stars', Ap. J., 158.

Underhill, A. B. 1970, in L. Houziaux and H. E. Butler, (Eds.), 'Ultraviolet Stellar Spectra and Related Ground-Based Observations', IAU Symp. 36, 215.

Underhill, A. B. 1971, 'Observing Programs for Early Type Stars with Extended Atmospheres', ESO Bull., No. 8 .

\section{b. Colors, models - theoretical}

Auer, L. H., Mihalas, D. 1969, 'NonLTE Model Atmospheres, III. A Complete Linearization Method', Ap. J., 158.

Auer, L. H. Mihalas, D. 1970, 'NonLTE Model Atmospheres. IV. Results for Multi-Line Computations', Ap. J., 160.

Auer, L. H., Mihalas, D. 1972, 'NonLTE Model Atmospheres VII. The Hydrogen and Helium Spectra of the O Stars', Ap. J. Suppl., 24, 193.

Canfield, R. C. $\quad 1971$, 'Deviations from Local Thermodynamic Equilibrium in Weak Complex Spectra', Astr. Ap., 10, 54.

Gebbie, K. B., Thomas, R. N. 1970, 'On the Dependence of $T_{\mathrm{e}}$, Upon Quantity Versus Quality of the Radiation Field in a Stellar Atmosphere', $A p . J ., 161,229$.

Gebbie, K. B., Thomas, R. N. 1971, 'The Temperature Control Bracket', Ap. J., 168, 461.

Grenfell, T. C. 1972, 'Model Atmospheres of White Dwarfs with Convection', Astr. Ap., $20,293$.

Gros, M. 1972, IAU Coll., No. 19, Greenbelt, Md.

Jennings, M. C., Dyck, H. M. 1972, 'The Consequences of Grains in the Atmospheres of Late-Type Stars. I. Intrinsic Polarization, Infrared Excesses, and Emission Lines', Ap. J., 177, 427.

Mihalas, D., Auer, L. H. 1970, 'NonLTE Model Atmospheres V. Multi-Line Hydrogen Helium Models for $\mathrm{O}$ and Early B Stars', Ap. J., 160.

Mihalas, D., Luebke, W. R. 1971, 'A NonLTE Picket-Fence Model in Radiative Equilibrium', M.N.R.A.S., 153, 229.

Mihalas, D. 1972, 'NonLTE Effects on Continuum and Hydrogen-Line Parameters in B and O Stars', Ap. J., 176, 139.

Peterson, D. M., Strom, S. E. 1969, 'Departures from LTE in the Hydrogen Lines of Late B Stars', Ap. J., 157.

Praderie, F. 1971, 'Some Remarks on $\mathrm{H}^{-}$in Stellar Atmospheres', Ap. J. Letters, 9, 27.

Price, M. J. 1969, 'A Relaxation Method for the Computation of Model Stellar Atmospheres', Ap. Space Sci., 4, 182.

Skumanich, A. $\quad 1970$, 'On the Existence of a Purely Radiative Temperature Rise in the Solar Chromosphere', Ap. J., 159.

Souffrin, P., Mihalas, D., Pagel, B. $\quad$ 1971, Théorie des atmosphères stellaires, Sauverny, Obser. de Genève. 


\section{c. Abundances, integrated line intensities - quasi-empirical}

Assousa, G. E., Smith, W. H. 1972, 'Radiative Lifetimes for Some Resonance Transitions of Fe $\mathrm{I}$ and $\mathrm{Fe}$ II in the Region Between $2300 \AA$ and $3050 \AA$, and the Application to Iron Abundance Determinations in the Sun and in the QSO PHL 938', Ap. J., 176, 259.

Conti, P. S. 1969, 'The Early A Stars IV. Analysis of the Double-Line Spectroscopic Binary ETA Virginis', Ap. J., 158.

Marlborough, J. M. 1971, 'A Comparison of Theoretical and Observational Results for Pleione's Shell Episode, 1938-1954', Ap. J., 163, 525.

O' Mara, B. J., Simpson, R. W. $\quad$ 1973, 'The Helium Abundance in 33 Main Sequence B Stars; Astr. Ap., (in press).

Sargent, W. L. W., Strom, K. M., Strom, S. E. 1969, 'An Analysis of the Peculiar A Star HD 204411', Ap. J., 157.

Smith, M. A. 1972, 'On Circumstellar Gas Emission Among Pre-Main-Sequence Stars in Ic Orionis and NGC 2264', Ap. J., 176, 617.

\section{d. Abundances, integrated line intensities - theoretical}

Athay, R. G., Lites, B. W. 1972, 'Fe I Ionization and Excitation Equilibrium in the Solar Atmosphere', Ap. J., 176, 809.

Hearn, A. G. $\quad$ 1970, 'The Abundance of Helium in Early-Type Stars and Departures from Local Thermodynamic Equilibrium', M.N.R.A.S., 150, 227.

Hearn, A. G. $\quad 1971$, 'Short Comm. Departures from Local Thermodynamic Equilibrium in the Neutral Helium Lines of Early Type Stars', M.N.R.A.S., 155.

Poland, A., Skumanich, A. $\quad 1971$, 'Non LTE Effects for Hel in Early B Type Stars', M.N.R.A.S., 152. Poland, A. I. $\quad 1972$, 'Helium in Hot Stars', Observatory, 92, 17.

Shipman, H. L., Strom, S. E. $\quad$ 1970, 'Lines of Neutral Helium in O- and B-Type Stars', Ap. J., 159.

Snyders, M. A. J., Underhill, A. B. 1971, 'Evidence Concerning the Complexity of the Line Forming Problem in He r', M.N.R.A.S., 151, 215.

Underhill, A. B. $\quad$ 1972, 'Helium in Hot Stars, Still a Problem', Observatory, 92, 18.

e. Line profiles, line effects, including blanketing - empirical

Worrall, G. 1971, 'The Doppler Core of the Solar Sodium D Lines', Astr. Ap., 12, 88.

f. Line profiles, line effects, including blanketing - quasi-empirical

Buscombe, W. 1969, 'Line Strengths for Southern OB Stars - I', M.N.R.A.S., 144, 1.

Delplace, A. M. 1970, 'Étude de l'instabilité de l'enveloppe de l'étoile Be HD $37202 \zeta$ Tauri', Astr. Ap., $7,68$.

Gabriel, A. H., Jordan, C. $\quad 1969$, 'Interpretation of Solar Helium-Like Ion Line Intensities', M.N.R.A.S., $145,241$.

Hearn, A. G. 1969, 'The Ultraviolet Resonance Lines of Neutral Helium From the Sun', M.N.R.A.S., $142,53$.

Hearn, A. G. 1969, 'A Suggestion for the Measurement of He II Line Intensities Emitted by the Sun', M.N.R.A.S., 142, 259.

Hearn, A. G., Noyes, R. W., Withbroe, G. L. 1969, 'The Differences Between Quiet and Active Regions Measured by Spectroheliograms in the Neutral Helium Resonance Lines', M.N.R.A.S., 144, 351.

Khokhlova, V. L. 1972, 'Helium Abundance Anomalies on the Surface of the Silicon Ap Star CU Virginis', Sov. Astr. - AJ, 15, No. 5.

Luud, L., Ilmas, M. 1971, 'Emission Lines in Stellar Spectra IV. Comparison of the Observed and Calculated Intensities of Hydrogen Lines', Tartu Astrophys. Obs. Teated, 32, 111.

Nguyen-Ngan 1970, 'Profile of the 10,830-Å Line on the Solar Disk', Sov. Astr. - AJ, 14, No. 2.

Nguyen-Ngan 1971 , '10 830 Line in Solar Active Regions', Sov. Astr. - AJ, 14, No. 4.

Schmidt, E. G. 1972, 'The Temperature Scale of F and G Stars I. Hydrogen Line Profiles, Ap. J., 174, 595.

Smith, L. F., Kuhi, L. V. 1970, 'Wolf-Rayet Stars. IV. Line Intensities in the Spectra of Two WN6 Stars', Ap. J., 162, 535 .

Underhill, A. B. $\quad$ 1970, 'The Hydrogen Lines in a B Type Supergiant', Astr. Ap., 6, 114. 
Viotti, R. 1970, 'Eta Carinae: Excitation of the Emission Lines and Some Considerations on Its Nature and the Observation of Ionized Metals Emission Lines in Stellar Spectra', Lab. di Astr. Frascati, Rapporto Interno 70/8.

g. Line profiles, line effects, including blanketing - theoretical

Altrock, R. C., Cannon, C. J., 1972, 'The Formation of Mg I 4571A in the Solar Atmosphere I. A Model Analysis of a One-Dimensional Static Atmosphere', Solar Phys. 26, 21.

Altrock, R. C., Cannon, C. J. 1973, 'The Formation of Mg I 4571A in the Solar Atmosphere II. The Effect of One-Dimensional Macroscopic Velocity Fields', Solar Phys., 29, 275.

Athay, R. G. 1970, 'Theoretical Line Intensities. IX Comment on the Effectively Thick Approximation', Ap. J., 161, 709.

Athay, R. G. $\quad 1970$, 'A NonLTE Line-Blanketed Solar Model', Ap. J., 161, 713.

Auer, L. H., Mihalas, D. 1973, 'Analyses of Light Ion Spectra in Stellar Atmospheres. IV. He I in the B-Stars', Ap. J., (submitted).

Brucato, R. J., Mihalas, D. 1971, 'On the Excitation Mechanism of N III Emission in the Of Stars', M.N.R.A.S., 154, 491.

Canfield, R. C. 1971, 'Formation of Solar Rare Earth Lines Inside and Outside H and K', Astr. Ap., 10,64 .

Castor, J. I. 1970, 'Spectral Line Formation in Wolf-Rayet Envelopes', M.N.R.A.S., 149, 111.

Castor, J. I., Smith, L. F., van Blerkom, D. 1970, 'Influence of Noncoherent Electron Scattering on a Line Profile in Wolf-Rayet Stars', Ap. J., 159, 1119.

Castor, J. I., Van Blerkom, D. 1970 , 'Excitation of He II in Wolf-Rayet Envelopes', Ap. J., 161, 485.

de Jager, C., Neven, L. $\quad 1973$, 'The Empirical Determination of Line Source Functions, $b_{\mathrm{L}}$-Values, and the Microturbulent and Convective Velocity Components as Functions of Depth in the PhotosphereChromosphere 'Transition Region', submitted to Comm. 36 files.

Dolginov, A. Z., Pavlov, G. G. 1972, Astronomich. Zhurnal., 49, No. 3.

Domke, H. 1971, Astrofizika, 7, No. 1.

Domke, H. 1971, Astrofizika, 7, No. 4.

Finn, G. D. 1973, 'Studies in Spectral Line Formation', submitted to Comm. 36 files.

Harrington, J. P. $\quad 1970$, 'On Schuster's Emission-Line Mechanism', Ap. J., 162, 913.

Ilmas, M. 1971, 'Emission Lines in Stellar Spectra II. Calculation of Some Transition Probabilities for Hydrogen', Tartu Astrophys. Obs. Teated, 32, 13.

Ilmas, M. 1971, 'Emission Lines in Stellar Spectra III. Calculation of the Hydrogen Spectrum', Tartu Astrophys. Obs. Teated, 32, 47.

Jones, H. P. 1971, 'The Formation of Resonance Lines in Multidimensional Media. I Scaling Properties in Two Dimensions', Ap. J., 164, 341.

Kafatos, M. C., Tucker, W. H. 1972, 'Time-Dependent Ionization Equilibrium and Line Radiation Under Flarelike Conditions', Ap. J., 175, 837.

Kamp, L. W. 1973, 'A NonLTE Study of Silicon Line Formation in Early-Type Main-Sequence Atmospheres', Ap. J., (submitted).

Lucy, L. B. 1971, 'The Formation of Resonance Lines in Extended and Expanding Atmospheres', Ap. $J ., 163,95$.

Luud, L., Ilmas, M. 1971, 'Emission Lines in Stellar Spectra I. The Formulation of the Problem', Tartu Astrophys. Obs. Teated, $32,3$.

Magnan, C. 1972, 'The Splitting of Lines in Differentially Rotating Slabs', Astr. Ap., (in press).

Mihalas, D. 1969, 'The Effects of Line Shifts on the Temperature Distribution in Stellar Atmospheres', Ap. J., 157.

Mihalas, D. 1972, 'Analyses of Light-Ion Spectra in Stellar Atmospheres I. Magnesium II in B and O Stars', Ap. J., 177, 115.

Mihalas, D. 1973, 'Analyses of Light-Ion Spectra in Stellar Atmospheres II. Ca In K Line in the B Stars', Ap. J., (submitted).

Mihalas, D., Athay, R. G. $\quad$ 1973, 'The Effects of Departures from LTE in Stellar Spectra', Ann. Rev. Astr. $A p$., (in press).

Mihalas, D., Hummer, D. G. $\quad$ 1973, 'Analyses of Light-Ion Spectra in Stellar Atmospheres. III. Nitrogen III in the O-Stars', $A p$. J., (submitted).

Mihalas, D., Lockwood, G. W. $\quad$ 1972, 'Observations of the He II $\lambda 10124$ Line in O and Of Stars', Ap. J., $175,757$.

Nussbaumer, H. $\quad$ 1971, 'N III and C III Emission in Of Stars', Ap. J., 170, 93. 
Panagia, N., Fulchignoni, M. $\quad 1970$, in L. Houziaux and H. E. Butler (eds.), 'Ultraviolet Stellar Spectra and Related Ground-Based Observations', IAU Symp. 36, 349.

Poland, A. I. $\quad 1970$, 'Neutral Helium Lines and the Helium Anomaly in Hot Stars', Ap. J., 160.

Rachkovski, D. N. 1971, Izvestia Crimean Astrophys. Obs., 43.

Simpson, R. W., O'Mara, B. J., Hearn, A. G. 1973, 'The Influence of Ultraviolet Line Blanketing on the Neutral Helium Triplet Lines in B-Type Stars', Astr. Ap., (in press).

Underhill, A. B. 1971, 'The Interpretation of Early-Type Spectra', Vistas in Astronomy 13, Ed. A. Beer, Pergamon Press, Oxford, p. 169.

Underhill, A. B., Hearn, A. G. 1971 , 'Some Aspects of the NonLTE Physics of the Helium Atom in Hot Stars', J.Q.S.R.T., 11, 617.

Vedmich, V. G. $\quad 1970$, Astrofizika, 6, No. 3.

Zinn, R. J. $\quad 1970$, 'The Temperature Dependence of $\mathrm{H} \beta$ Strength in O Stars', Ap. J., 162, 909.

\section{h. Inhomogeneities - quasi-empirical}

Pasachoff, J. M. 1971, 'The Calibration of the Wilson-Bappu Effect on the Sun', Ap. J., 164, 385.

\section{i. Inhomogeneities - theoretical}

Kandel, R. S. 1971, 'The Transfer of Lyman Continuum Radiation in Chromospheric Flares', Solar Phys., 21, 176.

\section{j. Polarization - quasi-empirical}

Pecker, J.-C. 1970, 'Some Considerations from the Direct Comparison Between the Observations and the Theory of Solar Disk Polarization', Solar Phys., 15, 88.

\section{k. Molecules - quasi-empirical}

Domke, H. $\quad$ 1971, Astronomich. Zhur., 48, No. 2.

Dumont, S. $\quad 1969$, 'Calcul du taux de polarisation du rayonnement continu', C. R. Acad. Sci. Paris, 268, 1978.

Dumont, S. 1971, 'Numerical Solution of the Transfer Equation for Polarized Continuum Radiation', J.Q.S.R.T., 11, 1675.

Dumont, S., Pecker, J.-C. 1971, 'Influence de l'absorption dans les raies sur la polarisation de spectre continu', Astr. Ap., 10, 118.

Gnedin, Yu. N., Dolginov, A. Z., Silant'ev, H. A. 1972, Astronomich. Zhur., 49, No. 4.

\section{Molecules - theoretical}

Praderie, F., Stecher, T. P. $\quad 1972$, 'On the Absorption by the $\mathrm{H}_{2}$ Molecule in UV Spectra', Astr. Ap., (in press).

m. Velocities, including hydromagnetics - theoretical

Caroff, L. J., Noerdlinger, P. D., Scargle, J. D. $\quad 1972$, 'Transfer of Resonance-Line Radiation in Differentially Expanding Atmospheres I. General Considerations and Monte Carlo Calculations', Ap. J., 176, 439.

Hillendhal, R. W. 1970, 'Observational Confirmation of a Mechanism for the Production of Extended Stellar Atmospheres', P.A.S.P., 82, 1231.

Limber, D. N. 1971, 'Asymmetries in the Envelopes of Wolf-Rayet Components of Close Binaries', Ap. $J ., 163,337$.

Noerdlinger, P. D., Scargle, J. D. $\quad$ 1972, 'Transfer of Resonance-Line Radiation in Differentially Expanding Atmospheres II. Analytic Solution for the Case of Coherence in the Frame of the Fluid', Ap. J., 176, 463.

n. Non-plane parallel, extended, end expanding atmospheres, including mass loss - quasi-empirical

Burlaga, L. F., Ogilvie, K. W. $\quad 1970$, 'Heating of the Solar Wind', Ap. J., 159.

Capps, R. W., Dyck, H. M. 1972, 'The Measurement of Polarized 10-Micron Radiation from Cool Stars with Circumstellar Shells', Ap. J., 175, 693.

Caputo, F., Viotti, R. $\quad$ 1970, 'On the Peculiar Spectrum of AG Carinae', Astr. Ap., 7, 266. 
Conti, P. S., Altschuler, W. R. Magnitudes', Ap. J., 170, 325.

Dyck, H. M., Forrest, W. J., Gillett, F. C., Stein, W. A., Gehrz, R. D., Woolf, N. J., Shaw1, S. J. 1971 , 'Visual Intrinsic Polarization and Infrared Excess of Cool Stars', Ap. J., 165, 57.

Galatola, A. 1972, 'Preliminary Analysis of the Atmospheric Eclipse of 32 Cygni', Ap. J., 175, 809.

Gammon, R. H., Gaustad, J. E., Treffers, R. R. 1972, 'Ten-Micron Spectroscopy of Circumstellar Shells', Ap. J., 175, 687.

Gillett, F. C., Merrill, K. M., Stein, W. A. 1971, 'Observations of Infrared Radiation from Cool Stars', Ap. J., 16483.

Hutchings, J. B. 1970, 'Expanding Atmospheres in OB Supergiants V. The Ultra-Violet Resonance Lines and Radiative Acceleration in the Orion Supergiants', M.N.R.A.S., 147, 367.

Hutchings, J. B. 1971, 'Rotationally Extended Stellar Envelopes II. HD 109378 ( $\kappa$ Dra) and 142926', M.N.R.A.S., 152, 109.

Hutchings, J. B., Wright, K. O. $\quad 1971$, 'Rotationally Extended Stellar Envelopes III. The Be Component of VV Cephei', M.N.R.A.S., 155, 203.

Morton, D. C. $\quad 1969$, 'Rocket Observations of Mass Loss from Hot Stars', Ap. Space Sci., 3, 117.

Nariai, K. 1973, 'La ligne d'H $\alpha$ en absorption déplacée au violet de l'étoile qui est depourvu d'hydrogène, HD 30353', Publ. Astr. Soc. Japan, (submitted).

Nugis, T. 1973, 'On the Chemical Composition of Wolf-Rayet Stars Envelopes', Publ. Tartu Astrophys. Obs', 40, (in press).

Nugis, T. 1973, 'On Temperatures of Wolf-Rayet Stars', Publ. Tartu Astrophys. Obs., 40, (in press).

Nugis, T. 1973, 'The Study of Emission Spectra of Wolf-Rayet Components of the Double Systems HD 193077 and HD 192641', Publ. Tartu Astrophys. Obs., 40, (in press).

Smith, L. F., Aller, L. H. 1971, 'A Detailed Comparison of the Spectra of a Planetary Nucleus and a Wolf-Rayet Star', Ap. J., 164, 275.

Underhill, A. B. $\quad$ 1969, 'The Wolf-Rayet Stars and Mass Loss', Ap. Space Sci., 3, 109.

Van Blerkom, D., Patton, G. (Yanchak) 1972, 'A Coarse Analysis of HD 50896', Ap. J., 177, 547.

Wilson, W. J., Schwartz, P. R., Neugebauer, G., Harvey, P. M., Becklin, E. E. 1972, 'Infrared Stars with Strong 1665/1667-MHz OH Microwave Emission', Ap. J., 177, 523.

o. Non-plane parallel, extended, and expanding atmospheres, including mass loss - theoretical

Borra, E. F., Fortier, L. 1972, 'A Search for Density and Pressure Inversions in High-Temperature, Low-Gravity Model Atmospheres', Ap. J., 177, 129.

Castor, J. I. 1970, 'Spectral Line Formation in Wolf-Rayet Envelopes', M.N.R.A.S., 149, 111.

Castor, J. I., Nussbaumer, H. 1971, 'On the Excitation of C III in Wolf-Rayet Envelopes', M.N.R.A.S., $155,293$.

Castor, J. I., Van Blerkom, D. $\quad 1970$, 'Excitation of He II in Wolf-Rayet Envelopes', Ap. J., 161, 485.

Cuperman, S., Harten, A. 1970, 'Noncollisional Coupling Between the Electron and the Proton Components in the Two-Fluid Model of the Solar Wind', Ap. J., 162, 315.

Cuperman, S., Harten, A. 1971, 'The Electron Temperature in the Two-Component Solar Wind', Ap. $J ., 163,383$.

Durney, B. R., Roberts, P. H. $\quad$ 1971, 'On the Theory of Stellar Winds', Ap. J., 170, 319.

Faulkner, D. J. $\quad 1970$, 'The Ejection of Planetary-Nebula Shells', Ap. J., 162, 513.

Gerola, H., Panagia, N. 1968, 'On the Spectrum of a Gaseous Nebula of Pure Hydrogen, I', Ap. Space Sci., $2,285$.

Huang, S. 1971, 'Transfer of Radiation in Circumstellar Dust Envelopes III. The Case of Distant Envelopes', Ap. J., 164, 91.

Kogure, T. 1969, 'On the Formation of the Balmer Line Spectrum in the Extended Atmospheres of Be Stars and Shell Stars', Publ. Astr. Soc. Japan, 21. No. 1.

Kutter, G. S. $\quad 1970$, 'Comments on Stellar Mass Loss', Ap. J., 160, 369.

Lucy, L. B., Solomon, P. M. $\quad 1970$, 'Mass Loss by Hot Stars', Ap. J., 159.

Marlborough, J. M., Roy, J.-R. $\quad$ 1970, 'Supersonic Stellar Winds in Early-Type Stars', Ap. J., 160.

Panagia, N. Ranieri, M. 1972, 'Lyman $\alpha$ Transfer in Dust-Filled Nebulae', I8th Liège Coll. on Planetary Nebulae, June.

Pecker, J.-C. 1970, 'Spectrum Formation in Stars with Steady-State Extended Atmospheres', IAU Coll. No. 2. Comm. 36, Munich, 1969, concluding remarks, NBS Special Publ., 332, 323.

Pecker, J.-C. 1971, 'Processus Physiques Intéressant les Grains de Poussière Circumstellaires: Charge et Effet Photoeléctrique', C.R. Acad. Sci., Paris, 272, 69. 
Pecker, J.-C. 1971, 'Poussières dans l'univers', Sciences, No. 74-75, 2.

Pecker, J.-C. 1971, 'Observation théorie et prédiction concernant les spectres infrarouge et radio des étoiles des premiers types', 17th Int'l. Astrophys. Symposium, Liege, Juin.

Pecker, J.-C. 1972, 'Evolution galactique I. Effets différentiels de la pression de radiation des étoiles des différents types sur les atomes neutres et les grains de poussière', Astr. Ap., 18, 253.

Pecker, J.-C. 1972, 'Processus Physiques Intéressant les Grains de Poussière Circumstellaires: II. Masse des grains et accrétion protonique', C. $R$. Acad. Sci., Paris, B274, 1001.

Pustylnik, I. 1971, 'Radiative Transfer in Atmospheres of Algol-Type Binaries I', Tartu Astr. Obs. Teated, $35,3$.

Simonneau, E. 1972 'Sur une nouvelle méthode pour l'étude de la formation des raies spectrales quand la fonction-source est indepéndante de la frequence', Compt. Rendus, 274, Serie B, 85.

Simmoneau, E. 1972, 'Résolution de équation de transfert en présence d'un champ de vitesses', Compt. Rendus, 275, Serie B, 169.

Sofia, S. $\quad$ 1969, 'Dynamical Study of an H II Region', P.A.S.P., 81, 265.

Van Blerkom, D., Castor, J. I. 1969, 'A Comment on the Method of Bappu and Ganesh for the Determination of Electron Temperatures of Wolf-Rayet Envelopes', M.N.R.A.S., 143, 461.

Wilson, R. E. $\quad 1971$, 'A Model of Epsilon Aurigae', Ap. J., 170, 529.

\section{Chromospheres}

a. Models-quasi-empirical

Doherty, L. R. 1970, 'OAO Observations of Mg in Emission in Late-Type Stars', Royal Soc., London, Mtg. on Solar Studies with Special Ref. to Space Observations.

Dumont, S. 1969, 'On the Interpretation of the Center-Limb Observations of Ca II Emission Cores', Astr. Ap., 2, 45.

Praderie, F. 1972, 'Evidence for Stellar Chromospheres Presented by Ground-Based Spectra of the Sun and Stars', IAU Coll., No. 19, Greenbelt, Md.

Simon, G. W., Noyes, R. W. 1971, 'Observed Heights of EUV Lines Formed in the Transition Zone and Corona', Harvard College Observatory, TR-27.

Tanaka, K., Hiei, E. 1972, 'The Chromospheric Continuum Observed at the Total Solar Eclipse of 12 November 1966 and a Model of the Low Chromosphere', Publ. Astr. Soc. Japan, 24, 323.

Weart, S. $\quad 1973$, 'The Photosphere-Chromosphere Transition: New Eclipse Data', submitted to Comm. 36 files.

Wilson, O. C. 1970, 'Dependence of Chromospheric Emission Upon Bolometric Luminosity for the Hyades', Ap. J., 160.

b. Models - theoretical

Reimers, D. 1971, 'Heat Conduction in the Solar Transition Layer and the Relation Between Temperature and Density of Coronal Active Regions', Astr. Ap., 10, 182.

Reimers, D. 1971, 'On the Solar Transition Layer and Solar Active Regions', Astr. Ap., 14, 198.

Ulmschneider, P. 1971, 'On the Computation of Shock Heated Models for the Solar Chromosphere and Corona', Astr. Ap., 12, 297.

c. Energy transfer, including velocities and hydromagnetics - quasi-empirical

Ulmschneider, P. 1970, 'Thermal Conductivity in Stellar Atmospheres I. Without Magnetic Field', Astr. Ap., 4, 144.

d. Energy transfer, including velocities and hydromagnetics - theoretical

Brengauz, V. D. 1971, 'Possible Nature of Short-Period Pulsations in the Solar Atmosphere', Sov. Astr. $A J, 15$, No. 3.

Frisch, H. 1971, 'Non-linear Coupling Between Thermal Conduction and Radiative Transfer', Astr. Ap., 13, 359.

Hearn, A. G, 1973, 'Chromospheric Heating of Very Hot Stars by Radiation Driven Sound Waves', Astr. Ap., (in press).

Hearn, A. G. 1973, 'Chromospheric Heating of Very Hot Stars by Radiation Driven Sound Waves II', Astr. Ap., (submitted). 
Jordan, S. 1970, 'The Effect of the Density Scale Height on Shock-Wave Heating in the Low Solar Chromosphere', Ap., J., 161, 1189.

Kaburaki, O., Uchida, Y. 1971, 'Magnetohydrodynamic Wave-Mode Coupling. Quantum FieldTheoretical Approach to Weakly Non-Linear Case with Application to Solar Coronal Heating', Publ. Astr. Soc. Japan, 23, 405.

Lerche, J. 1971, 'Resistive Effects in the Turbulent Photosphere and Chromosphere', Ap. Space Sci., 10, 486.

Mullan, D. J. 1971, 'The Structure of Transverse Hydromagnetic Shocks in Regions of Low Ionization', M.N.R.A.S., 153, 145 .

Nguyen-Ngan 1970, 'The Energy Balance in Spicules', Sov. Astr. - AJ, 14, No. 1.

Pikel'ner, S. B. $\quad 1969$, ‘A Mechanism for the Formation of Chromospheric Spicules', Sov. Astr. - AJ, 13, No. 2.

Praderie, F., Thomas, R. N. 1972, 'On Estimates of the Nonradiative Energy Input to the Solar Chromosphere from the $\mathrm{H}^{-}$Emission', Ap. J., 172, 485.

Saito, M. 1970, 'An Interpretation of the Observed Chromospheric Radial Velocities of the K-Type Component of Zeta Aurigae', Publ. Astr. Soc. Japan, 22, 455.

Uchida, Y. 1969, 'A Mechanism for the Acceleration of Solar Chromospheric Spicules', Publ. Astr. Soc. Japan, 21, No. 2.

Ulmschneider, P. 1971, 'On the Propagation of a Spectrum of Acoustic Waves in the Solar Atmosphere', Astr. Ap., 14, 275.

Worrall, G. 1972, 'Oscillations in an Isothermal Atmosphere: The Solar Five-Minute Oscillations', $A p$. $J ., 172,749$.

e. Excitation state - quasi-empirical

Athay, R. G. 1970, 'The Chromospheric Abundance of Iron', Astr. Ap., 7, 305.

Ishizawa, T. 1971, 'Emission-Line Intensities of the Ca II Atom from a Finite Atmosphere', Publ. Astr. Soc. Japan, 23, 75.

Pecker, J.-C., Pottasch, S. R. $\quad 1969$, 'On the Abundance Determination in the Solar Chromosphere', Astr. Ap., $2,81$.

Tanaka, K. 1971, 'Metallic Lines in the Lower Chromosphere Observed at Peruvian Eclipse I. Observational Results', Publ. Astr. Soc. Japan, 23, 185.

Tandberg-Hanssen, E., Smythe, C. $\quad 1970$, 'The Excitation of Ionized Barium in the Chromosphere', $A p$. $J ., 161,289$.

\section{f. Inhomogeneities - quasi-empirical}

Bappu, M. K. V. Sivaraman, K. R. 1971, 'K Emission-Line Widths and the Solar Chromosphere', Solar Phys., 17, 316.

Vernazza, J. E., Noyes, R. W. 1971, 'Inhomogeneous Structure of the Solar Chromosphere from LymanContinuum Data', Harvard College Observatory, TR-25.

\section{g. Inhomogeneities - theoretical}

Beebe, H. A., Johnson, H. R. $\quad$ 1972, 'Ca II K Emission Arches', Solar Phys., $27,34$.

Brengauz, V. D. 1969, 'The Problem of the Existence of Short-Period Pulsations of the Magnetic Field in the Solar Atmosphere', Sov. Astr. - AJ, 13, No. 2.

\section{Coronas}

\section{a. Models - theoretical}

Beigman, I. L., Vainshtein, L. A. 1971, 'Thermal Model of Solar Corona Active Regions', Sov. Astr. $A J, 14$, No. 5.

Blumenthal, G. R., Drake, G. W. F., Tucker, W. H. 1972, 'Ratio of Line Intensities in Helium-Like Ions as a Density Indicator', $A p . J ., 172,205$.

De Loore, C. 1970, 'Convection Regions and Coronas of Sun and Stars', Ap. Space Sci., 6, 60.

De Jager, C., De Loore, C. 1971, 'Coronae of Cool Stars', Ap. Space Sci., 11, 284.

Illarionov, A. F., Syunyaev, R. A. 1970, 'Thomson Scattering as a Cooling Mechanism for the Solar Corona', Sov. Astr. - AJ, 14, No. 5. 
Jefferies, J. T., Orrall, F. Q., Zirker, J. B. $\quad$ 1972, 'The Interpretation of Total Line Intensities from Optically Thin Gases I. A General Method', Solar Phys., 22, 307.

Jefferies, J. T., Orrall, F. Q., Zirker, J. B. 1972, 'The Interpretation of Total Line Intensities from Optically Thin Gases II. The Coronal Forbidden Lines', Solar Phys., 22, 317.

Jefferies, J. T., Orrall, F. Q., Zirker, J. B. $\quad$ 1972, 'The Interpretation of Total Line Intensities from Optically Thin Gases. III. Application to Coronal Forbidden Line Spectra', Solar Phys., 22, 327.

Nariai, K. 1969, 'Coronae Around Helium Stars and X-Ray Sources', Ap. Space Sci., 3, 160. Vil'koviskii, É. Ya., Obashev, S. O. 1972, 'Emissivity and Temperature Structure of the Coronal Plasma', Sov. Astr. - AJ, 15, No. 4.

\section{b. Excitation state - theoretical}

Tanaka, K. 1971, 'Metallic Lines in the Lower Chromosphere Observed at Peruvian Eclipse II. Interpretation of Chromospheric Excitations by Multi-Level Line Transfer', Publ. Astr. Soc. Japan, 23, 217.

\section{c. Mass flux - theoretical}

Belcher, J. W. 1971, 'Alfvénic Wave Pressure and the Solar Wind', Ap. J., 168, 509.

Durney, B. R., Werner, N. 1972, 'On the Domains of Existence of the Three Types of Supersonic Solutions of the Inviscid Solar-Wind Equations', Ap. J., 171, 609.

Durney, B. $\quad$ 1972, 'Polytropic Subsonic Stellar Winds with Magnetic Fields', Ap. Space Sci, 17, 489.

Grzedzielski, S. 1969, 'Solar-Wind Model Including the Effects of Rotation, Magnetic Fields, and Anisotropic Heat Conduction', Ap. Space Sci., 3, 139.

Nariai, K. 1969, 'Mass Loss from Coronae and Its Effect Upon Stellar Rotation', Ap. Space Sci., 3, 150.

Unsold, A. $\quad$ 1970, 'Solar Corona, Interplanetary Plasma and Prominences', Astr. Ap., 4, 220.

Weidelt, R. D. 1970 , 'Stellar Wind for Non-negligible Gravitational Potential of the Atmosphere', $A p$. Space Sci., 6, 205.

Whang, Y. C. 1971, 'Conversion of Magnetic-Field Energy Into Kinetic Energy in the Solar Wind', Ap. $J ., 169,369$.

\section{Circumstellar shells, nebulae, and non-coronal mass fux}

\section{a. Quasi-empirical}

Ade, P., Pagel, B. E. J. $\quad 1970$, 'Reddening of $\eta$ Carinae from Permitted Fe n Lines', Observatory, 90, 6.

Aller, L. H., Czyzak, S. J. $\quad 1970$, 'Spectrophotometric Studies of Gaseous Nebulae XVI. The Moderately High-excitation Planetaries IC 2003, NGC 2022, and CD-23 ${ }^{\circ} 12238^{\prime}, A p . J ., 160$.

Aller, L. H., Czyzak, S. J., Buerger, E. G., Lee, P. 1972, 'Spectrophotometric Studies of Gaseous Nebulae XX. The Inhomogeneous, Low Excitation, Planetary NGC 40', Ap. J., 172, 361.

Burbidge, G. R., Stein, W. A. 1970, 'Cosmic Sources of Infrared Radiation', Ap. J., 160.

Czyzak, S. J., Aller, L. H., Kaler, J. B. $\quad$ 1971, 'Spectrophotometric Studies of Gaseous Nebulae XIX. The Moderate-Excitation Planetary NGC 6826', Ap. J., 168, 405.

Davidson, K. 1971, 'On the Nature of Eta Carinae', M.N.R.A.S., 154, 415.

Faraggiana, R., Hack, M. 1968, 'Spectroscopic Evidence for Mass Loss from CH Cygni', Ap. Space Sci., $3,205$.

Gehrz, R. D., Woolf, N. J. $\quad$ 1971, 'Mass Loss from M Stars', Ap. J., 165, 285.

Goad, L. E., Goldberg, L., Greenstein, J. L. $\quad 1972$, 'High- $n$ Balmer Transitions in Gaseous Nebulae', $A p$. $J ., 175,117$.

Kaler, J. B. $\quad 1970$, 'Chemical Abundances and the Parameters of Planetary Nebulae', Ap. J., 160.

Krueger, T. K., Aller, L. H., Czyzak, S. J. $\quad$ 1970, 'Some Forbidden-Line Intensity Ratios in Gaseous Nebulae', Ap. J., 160.

Low, F. J., Johnson, H. L., Kleinmann, D. E., Latham, A. S., Geisel, S. L. $\quad$ 1970, 'Photometric and Spectroscopic Observations of Infrared Stars', Ap. J., 160.

Mathis, J. S. $\quad$ 1970, 'Internal Dust in Gaseous Nebulae', Ap. J., 159.

Miller, J. S., Mathews, W. G. $\quad$ 1972, 'The Recombination Spectrum of the Planetary Nebula NGC 7027', Ap. J., $172,593$.

Nordsieck, K. H. 1971, 'A Further Comment on the Dust-Radiation Instability in Ionized Nebulae', Ap. J., $166,731$.

O'Dell, C. R., Terzian, Y. $\quad$ 1970, 'The Planetary Nebula BD +30³639', Ap. J., 160. 
Peimbert, M., Torres-Peimbert, S. $\quad$ 1971, 'Planetary Nebulae III. Chemical Abundances', Ap. J., $168,413$. Perinotto, M. $\quad$ 1971, 'Temperature and Density in Gaseous Nebulae. II', Astr. Ap., 14, 78.

Saraph, H. E., Seaton, M. J. 1970, 'Electron Densities in Planetary Nebulae', M.N.R.A.S., $148,367$.

Strom, K. M., Strom, S. E., Yost, J. 1971, 'Circumstellar Shells in the Young Cluster NGC 2264 ', Ap. $J ., 165,479$.

Strom, S. E., Strom, K. M., Brooke, A. L., Bregman, J., Yost, J. 1972, 'Circumstellar Shells in the Young Cluster NGC 2264 II. Infrared and Further Optical Observations', Ap. J., 171, 267.

Strom, S. E., Strom, K. M., Yost, J., Carrasco, L., Grasdalen, G. 1972, 'The Nature of the Herbig Aeand Be-Type Stars: Associated with Nebulosity', Ap. J., 173, 353.

Swamy, K. S. K. $\quad$ 1971, 'On the Infrared Radiation from Eta Carinae', Observatory, 91, 120.

Swamy, K. S. K. $\quad$ 1972, 'Dust Models for R Coronae Borealis and RY Sagittarii', P.A.S.P., 84.

Thomasson, P., Davies, J. G. 1970, 'Further Radio Observations of Planetary Nebulae', M.N.R.A.S., $150,359$.

Van Blerkom, D. J. $\quad$ 1971, 'Physical Parameters of Two Planetary Nebulae with Wolf-Rayet Nuclei', $A p$. $J ., 166,343$.

van Rensbergen, W., Wuyts, J. $\quad 1970$, 'On the Population of the $2^{3} S$ Level of He in Planetary Nebulae', Astr. Ap., 9, 325.

Wallerstein, G. 1971, 'On the Infrared Excess of W Cephei and Similar Stars', Ap. J., 166, 725.

\section{b. Theoretical}

Avedisova, V. S. $\quad$ 1972, 'Formation of Nebulae by Wolf-Rayet Stars', Sov. Astr. - AJ, 15, No. 5.

Bahcall, J. N., Kozlovsky, B.-Z., Salpeter, E. E. 1972, 'On the Time Dependence of Emission-Line Strengths from a Photoionized Nebula', Ap. J., 171, 467.

Brocklehurst, M. 1970, 'Level Populations of Hydrogen in Gaseous Nebulae', M.N.R.A.S., $148,417$.

Brocklehurst, M. 1971, 'Calculations of the Level Populations for the Low Levels of Hydrogenic Ions in Gaseous Nebulae', M.N.R.A.S., 153, 471.

Brocklehurst, M. 1972, 'The Line Spectra of Helium in Gaseous Nebulae', M.N.R.A.S., $157,211$.

Capriotti, E. 1971, 'The Formation of Condensations in Planetary Nebulae I', Ap. J., 166, 563.

Cox, D. P., Daltabuit, E. 1971, 'Effects of Collisional Excitation on the Intensities of the $5876 \AA$ and 4471 Å Lines of Neutral Helium', Ap. J., 167, 257.

Drake, G. W. F., Robbins, R. R. 1972, 'The Population of Helium Triplet States in Gaseous Nebulae', Ap. J., 171, 55.

Finzi, A., Wolf, R. A. 1971, 'Ejection of Mass by Radiation Pressure in Planetary Nebulae', Astr. Ap., 11, 418.

Fix, J. D. 1969, 'The Formation and Growth of Carbon Particles in the Atmospheres of Cool Carbon Stars', M.N.R.A.S., 146, 37.

Fix, J. D. 1969, 'The Formation and Growth of Carbon Particles in the Atmospheres of Mira Variables', M.N.R.A.S., 146, 51 .

Flower, D. R. 1969, 'The Ionization Structure of Planetary Nebulae VII. The Heavy Elements', M.N.R.A.S., 146, 171 .

Flower, D. R. 1969, 'The Ionization Structure of Planetary Nebulae VIII. Models of the Nebulae NGC7662 and IC 418', M.N.R.A.S., 146, 243.

Flower, D. R. $\quad 1970$, 'On the Infrared Line Spectra of Planetary Nebulae', M.N.R.A.S., $147,245$.

Hunter, J. H., Sofia, S. 1971, 'The Dynamics and Thermal Stability of Planetary Nebulae', M.N.R.A.S., 154, 393.

Isobe, S. 1971, 'Evaporation of Dirty Ice Particles Surrounding Early Type Stars II. The Orion Association', Publ. Astr. Soc. Japan. 23, 371.

Kaler, J. B. 1972, 'Excitation of Nebular Spectrum Lines', Ap. J., 173, 601.

Kostyakova, E. B. 1970, 'Investigation of Planetary Nebulae in the Near Ultraviolet Region of the Spectrum', Sov. Astr. - $A J, 14$, No. 5.

Kovach, W. S. 1971, 'The Dynamics and Infrared Radiation of Young, Dust-Filled Planetary Nebulae', Ap. J., 168, 423.

Leibowitz, E. M. 1972, 'The Emission Spectrum of the Ion C Iv in Planetary Nebulae', M.N.R.A.S., 157, 97.

Leibowitz, E. M. 1972, 'Polarization of C rv Emission Lines in Planetary Nebulae', M.N.R.A.S., 157, 115.

Mathis, J. S. 1971, 'Internal Dust in Gaseous Nebulae II. Absorption of Lyman-Continuum Radiation by Dust', $A p . J ., 167,261$. 
Perinotto, M. 1971, 'Temperature and Density in Gaseous Nebulae', Astr. Ap., $10,421$.

Robbins, R. R., Robinson, E. L. 1971, 'Capture-Cascade Intensities of the Helium Singlets in Nebulae', Ap. J., 167, 249.

Seaton, M. J. $\quad 1969$, 'Excitation of High Balmer Lines: Case C of Aller, Baker, and Menzel M.N.R.A.S., $145,91$.

Swamy, K. S. K., Stecher, T. P. $\quad 1969$, 'On the Formation of Planetary Nebulae', P.A.S.P., 81, 873.

Swamy, K. S. K. $\quad 1970$, 'Circumstellar Dust Model for Infrared Stars', Ap. Space Sci, 9, 123.

Van Blerkom, D. $\quad 1969$, 'Radiative Excitation in Planetary Nebulae', M.N.R.A.S., 145, 75.

Yankulova, I. M. 1969, 'A Model for the Fine Radial Filaments Observed in the Planetary Nebula NGC 7293', Sov. Astr. - AJ, 13, No. 1.

\section{Statistical thermodynamics, radiative transfer, and aerodynamics}

\section{a. Theoretical}

Adams, T. F. 1971, 'The Effect of Recoil in Resonance-Line Scattering', Ap. J., 168, 575.

Adams, T. F. $\quad$ 1972, 'The Escape of Resonance-Line Radiation from Extremely Opaque Media', Ap. J., 174, 439 .

Anderson, J. L., Spiegel, E. A. 1972, 'The Moment Method in Relativistic Radiative Transfer', $A p . J$. , $171,127$.

Athay, R. G. 1972, 'A Probabilistic Formulation of the Noncoherent-Scattering Problem', Ap. J., 176, 659.

Auer, L. H., Mihalas, D. 1970, 'On the Use of Variable Eddington Factors in NonLTE Stellar Atmospheres Computations', M.N.R.A.S., 149, 65.

Barcza, S. $\quad$ 1971, 'Highly Excited Hydrogen Lines in Stellar Spectra I', Ap. Space Sci., 13, 36.

Busse, F. H. 1970, 'Differential Rotation in Stellar Convection Zones', Ap. J., 159.

Cannon, C. J. 1970, 'Line Transfer in Two Dimensions', Ap. J., 161, 255.

Cannon, C. J., Rees, D. E. 1971, 'Line Transfer in the Presence of Two-Dimensional Velocity Gradients', Ap. J., 169, 157.

Carson, T. R. $\quad 1969$, 'Ionization Equilibrium with Negative Ion Formation', M.N.R.A.S., 142, 409.

Cassinelli, J. P., Hummer, D. G. 1971, 'Radiative Transfer in Spherically Symmetric Systems II. The Non-Conservative Case and Linearly Polarized Radiation', M.N.R.A.S., 153, 9.

Code, A. D. $\quad$ 1970, 'A Solution of a Time-Dependent Equation of Radiative Transfer', Ap. J., 159.

Code, A. D., Eason, G. $\quad 1970$, 'Time-Dependent Radiation Transfer in a Semi-Infinite Atmosphere', $A p$. J., 159.

Collins, G. W. II 1972, 'Transfer of Polarized Radiation in a Stellar Atmosphere', Ap. J., $175,147$.

Cox, D. P., Daltabuit, E. $\quad 1971$, 'Radiative Cooling of a Low-Density Plasma', $A p . J ., 167,113$.

Culhane, J. L. $\quad$ 1969, 'Thermal Continuum Radiation from Coronal Plasmas at Soft X-Ray Wavelengths', M.N.R.A.S., 144, 375.

Delache, Ph., Foeschle, Ch. 1972, 'Time Dependent Radiative Transfer. Damping of a Temperature Fluctuation I. Near LTE Grey Approximation', Astr. Ap., 16, 348.

Doschek, G. A., Donahue, T. M. 1970, 'Transport of Resonance Radiation in Optically Thick Media', Ap. J., 161, 737.

Faÿ, T., Marenin, I., Van Citters, W. 1900, 'Perturbation Analysis and Constants for the Red System of the Cyanide Radical', Goethe Link Observatory, Indiana University, No. 119.

Finn, G. D. 1973, 'Probabilistic Radiative Transfer', submitted to Comm. 36 files.

Fischel, D., Sparks, W. M. 1971, 'Partition Functions in Ionizing Plasmas', Ap. J., $164,359$.

Fuller, F. B., Warming, R. F. 1970, 'An Invariant Imbedding Approach to Spectral-Line Formation by Noncoherent Scattering', Ap. Space Sci., 7, 93.

Grasboske, H. C. Jr. 1972, 'Equilibrium Thermodynamics of Nonideal Reacting Gases', Ap. J., 172, 689.

Gruschinske, J., Ueno, S. 1970, 'Bellman's New Approach to the Numerical Solution of the Auxiliary Equation in a Spherical Medium', Publ. Astr. Soc. Japan, 22, 365.

Hasimoto, Y. 1972, 'Time Development of Axisymmetric Shock', Progress of Theoretical Physics, $47,83$.

Hummer, D. G. 1969, 'Non-coherent Scattering VI. Solutions of the Transfer Problem with a Frequency Dependent Source Function', M.N.R.A.S., 145, 95.

Hummer, D. G., Rybicki, G. B. 1970, 'Non-coherent Scattering VII. Frequency Dependent Thermalization Lengths and Scattering with Continuous Absorption', M.N.R.A.S., 150, 419. 
Hummer, D. G., Rybicki, G. B. 1971, 'Radiative Transfer in Spherically Symmetric Systems: The Conservative Grey Case', M.N.R.A.S., 152, 1.

Ishizawa, T. 1971, 'The Effect of External Radiation on Emission-Line Intensity', Publ. Astr. Soc. Japan, $23,121$.

Ivanov, V. V. $\quad 1970$, Astrofizika, 6, No. 4.

Ivanov, V. V. 1972, Astronomich. Zhur., 49, No. 1.

Ivanov, V. V., Sabashvili, Sh. A. 1972, 'Transfer of Resonance Radiation and Photon Random Walks', Ap. Space Sci., 17, 13.

Jefferies, J. T. 1971, 'Population Inversion in the Outer Layers of a Radiating Gas', Astr. Ap., 12, 351.

Johnson, H. R., Marenin, I. R. 1973, 'Radiative Opacity Due to the Red System of CN', submitted to Comm 36 files.

Jordan, C. 1969, 'The Ionization Equilibrium of Elements Between Carbon and Nickel', M.N.R.A.S., 142,501 .

Jordan, C. 1970, 'Ionization Equilibria for High Ions of Fe and Ni', M.N.R.A.S., 148, 17.

Kemp, J. C. 1970, 'Circular Polarization of Thermal Radiation in a Magnetic Field', Ap. J., 162, 169.

Kho, T. H., Sen, K. K. 1971, 'On Combined Operational Method for Transfer Problems in Homogeneous, Spherical Media', Ap. Space Sci., 14, 223.

Kulander, J. L. 1971, 'The Velocity-Dependent Non LTE Source Function II. Finite Layers', Ap. J., 165. 543.

Lerche, I. 1971, 'Turbulent Velocity Waves and Kinematic Dynamo Activity', Ap. Space Sci., 13, 137.

Matsumoto, M. 1968, 'The Solution of Milne's Problem by Duhamel's Principle', Publ. Astr. Soc. Japan, 20, No. 2.

McCormick, N. J., Siewert, C. E. $\quad 1970$, 'Spectral-Line Formation by Noncoherent Scattering', Ap. J., $162,633$.

Mihalas, D. 1971, 'A Comment on the Interpretation of the Broad Component of N III $\lambda \lambda 4634-4640$ Emission in Of Stars', $A p . J ., 170,541$.

Minin, I. N. $\quad 1971$, Astronmich. Zhur., 48, No. 2.

Minin, I. N. 1971, Vestnik of the Leningrad Univ., No. 7.

Monagham, J. J. $\quad 1970$, 'A Perturbation Solution of the Milne Problem', M.N.R.A.S., 148, 353.

Nagirner, D. I. $\quad 1972$, Astrofizika, 8, No. 3.

Natta, A., Ranieri, M. 1972, 'Statistics on the Solar Spectrum Suitable for the Study of the Blanketing Effect in Stars of Spectral Types F, G and K', Ap. Space Sci., 17, 390.

Omont, A., Smith, E. W., Cooper, J. 1972, 'Redistribution of Resonance Radiation I. The Effect of Collisions', Ap. J., 175, 185. .

Pustylnik, I. 1971, 'Radiative Transfer in Atmospheres of Close Binaries', Publ. Tartu Astrophys. Obs.,

Rybicki, G. B., Hummer, D. G. 1968, 'Non coherent Scattering V. Thermalization Distances and Their Distribution Function', M.N.R.A.S., 144, 313.

Sapar, A. 1973, 'Simple Method to Solve The Equation of Radiative Transfer', Publ. Tartu Astrophys. Obs. 40.

Sarychev, A. P. 1970, 'The Applicability of the Boltzmann Distribution for the Vibrational States of Molecules on the Sun', Sov. Astr. - AJ, 14, No. 6.

Shimooda, H. 1972, 'Analytical Solutions of Jefferies' Equations for the Grand Source Functions', Publ. Astr. Soc. Japan, 24, 287.

Siewert, C. E., Ozisik, M. N. 1969, 'An Exact Solution in the Theory of Line Formation', M.N.R.A.S., 146, 351.

Skalafuris, A. J. 1969, 'The Structure of Shock Front in Atomic Hydrogen', Ap. Space Sci., 3, 234.

Sobolev, V. V. $\quad 1970$, Astronomich. Zhur., 47, No. 2.

Sobolev, V. V. 1972, Astrofizika, 6, No. 4.

Sparks, W. M., Fischel, D. 1971, 'Partition Functions and Equations of State in Plasmas', NASA-SP 3066.

Sweigart, A. V. 1970, 'Radiative Transfer in Atmospheres Scattering According to the Rayleigh Phase Function with Absorption', Ap. J. Suppl., 22, 1.

Tarafdar, S. P., Vardya, M. S. 1970, 'Absorption Coefficient of $\mathrm{H}^{-}$and Interparticle Interaction I. Debye-Huckel Approximation', J.Q.S.R.T., 10.

Tarafdar, S. P., Vardya, M. S. 1970, 'Absorption Coefficient of $\mathrm{H}^{-}$and Interparticle Interaction II. Forbidden Bound-Free Continuum', M.N.R.A.S., 150, 9.

Tarafdar, S. P., Vardya, M. S. 1972, 'Missing Solar Ultraviolet Opacity and Diatomic Molecules', Ap. $J ., 171,185$. 
Tarafdar, S. P., Vardya, M. S. 1973, 'On Ultraviolet Stellar Fluxes IV. Importance of Bound-Free Absorption of SI in B to K Stars', $A p . J ., 179$, (in press).

Ueno, S. 1971, 'Solution of the Restricted Two-level Line Formation Problem', Publ. Astr. Soc. Japan, 23, 239.

Ueno, S., Kagiwada, H., Kalaba, R. 1971, 'Radiative Transfer in Spherical Shell Atmospheres with Radial Symmetry', Journal of Math. Physics, $12,7$.

Vardya, M. S. 1970, 'Partition Functions and Equilibrium Constants for ScO, YO and LaO', Astr. Ap., $5,162$.

Vijk, T. 1970, 'Radiative Transfer in a Spherical Atmosphere of Free Electrons', Trans. Acad. Sci. Estonian S.S.R., 19, 184.

Vijk, T. $\quad$ 1970, 'On Solving the Spherical Transfer Equation', Trans. Acad. Sci. Estonian S.S.R., 19, 286.

Vijk, T. 1971, 'Solution of the Equation of Transfer for Spherical Atmosphere by the Method of Grant and Hunt', Publ. Tartu Astrophys. Obs., 39, 3.

Vijk, T. 1971, 'Solution of the Equation of Radiative Transfer by the Method of Regional Averaging', Publ. Tartu Astrophys. Obs., 39, 34.

Vijk, T. 1971, 'Method of Regional Averaging in the Radiative Transfer', Trans. Acad. Sci. Estonian S.S.R., 20, 285.

Virgopia, N., Vardya, M.S. $\quad 1971$,'Molecular Ratios Sensitive to Elemental Abundances', P.A.S.P., 83, 222.

Vityasev, V. V. $\quad 1970$, Astrofizika, 6, No. 3.

Vityasev, V. V. 1972 , Astrofizika, 8, No. 2.

Wildt, R. 1972, 'Thermodynamics of the Gray Atmosphere IV. Entropy Transfer and Production', Ap. $J ., 174,69$.

Yengibarian, N. B., Nikogossian, A. G. $\quad$ 1971, Astrofizika, 7, No. 4.

Yengibarian, N. B., Nikogossian, A. G. $\quad 1972$, Astrofizika, 8, No. 1.

Yengibarian, N. B., Nikogossian, A. G. $\quad$ 1972, Astrofizika, 8, No. 2.

\section{PART C: AIMS AND ACTIVITIES OF THE COMMISSION}

It has been our view that Commission 36 should serve as a center for the exchange of ideas and results on the structure of stellar atmospheres and for collaboration with other commissions in related fields. We believe, moreover, that it should serve this function not only for its formal members and for members of the IAU, but for all interested astrophysicists.

Toward these ends, we have:

(1) Established a class of associate membership, which includes all those who, for official reasons, either cannot or do not wish to become formal members. The list of members and associates is available on request.

(2) Established a center for the exchange of bibliographical material, for those members who wish to use it. The present bibliography is based in part on this material.

(3) Encouraged the organization of colloquia and symposia, of which the following were sponsored in part by the Commission.

'Wolf-Rayet and High Temperature Stars', IAU Symposium, No. 49, Buenos Aires, August 9-14, 1971. Chairman: M. K. V. Bappu.

'Stellar Chromospheres', IAU Colloquium, No. 19, Greenbelt, Maryland, February 14-18, 1972. Chairman: A. B. Underhill.

'Extended Atmospheres and Circumstellar Matter in Spectroscopic Binary Systems', IAU Symposium, No. 51 (Struve Memorial), Victoria, B. C., September 6-12, 1972. Chairman: K. O. Wright.

In addition, the following conferences, although not sponsored by Commission 36, dealt with topics of interest to its members:

'Line Formation in the Presence of Magnetic Fields', Boulder, August 3 - September 2, 1971. Chairman: R. G. Athay, L. House, and G. A. Newkirk.

'Supergiant Stars', Trieste, September 6-8, 1971. Chairman: M. Hack.

'Red Giant Stars', Bloomington, Indiana, October 4-6, 1972. Chairman: H. R. Johnson.

(4) Encouraged the quick and inexpensive publication of proceedings of current symposia, including the following: 
'Spectrum Formation in Stars with Steady-State Extended Atmospheres', Proceedings of IAU Colloquium No. 2, Eds. H. G. Groth and P. Wellman. NBS Spec. Publ. 332 (Washington, D.C.: U.S. Government Printing Office) 1970. Price $\$ 1.75$.

'The Menzel Symposium on Solar Physics, Atomic Spectra, and Gaseous Nebulae', Ed. K. B. Gebbie. NBS Spec. Publ. 353 (Washington, D. C.: U.S. Government Printing Office) 1971. Price $\$ 1.75$.

'Stellar Chromospheres', Proceedings of IAU Colloquium, No. 19, Eds. E. H. Avrett and S. D. Jordan. NASA SP-317 (Washington, D. C.: U.S. Government Printing Office) 1973.

'Line Formation in the Presence of Magnetic Fields', Eds. R. G. Athay, L. House, and G. A. Newkirk (Boulder: NCAR-HAO) 1971. Price free.

'Théorie des Atmosphères Stellaires', Eds. D. Mihalas, B. E. J. Pagel, and P. Souffrin (Geneva: Observatoire de Genève) 1972. Price $\$ 6.00$.

'Mass Loss From Stars', Proceedings of the Second Trieste Colloquium on Astrophysics, Ed. M. Hack (Dordrecht-Holland: D. Reidel Publishing Co.) 1969. Price $\$ 22.10$

In addition, the proceedings of the two IAU Symposia mentioned above are being published by D. Reidel Publishing Company, Dordrecht, at the standard IAU prices.

R. N. THOMAS

President of the Commission 